Revue des patrimoines

$31 \mid 2017$

Patrimoines de la santé : essais de définition - enjeux de conservation

\title{
Tuberculose et patrimoine : les établissements de Perharidy et du Laber à Roscoff (Finistère)
}

Tuberculosis and heritage: the Perharidy and Laber establishments in Roscoff (Finistère)

Christel Douard et avec la collaboration de Valérie Guesnier

\section{(2penEdition}

Journals

Édition électronique

URL : http://journals.openedition.org/insitu/14011

DOI : 10.4000/insitu. 14011

ISSN : 1630-7305

\section{Éditeur}

Ministère de la Culture

\section{Référence électronique}

Christel Douard et avec la collaboration de Valérie Guesnier, «Tuberculose et patrimoine : les établissements de Perharidy et du Laber à Roscoff (Finistère) », In Situ [En ligne], 31 | 2017, mis en ligne le 03 octobre 2017, consulté le 09 octobre 2020. URL : http://journals.openedition.org/insitu/ 14011 ; DOI : https://doi.org/10.4000/insitu.14011

Ce document a été généré automatiquement le 9 octobre 2020.

\section{c) (ㅇ)}

In Situ Revues des patrimoines est mis à disposition selon les termes de la licence Creative Commons Attribution - Pas d'Utilisation Commerciale - Pas de Modification 4.0 International. 


\section{Tuberculose et patrimoine : les établissements de Perharidy et du Laber à Roscoff (Finistère)}

Tuberculosis and heritage: the Perharidy and Laber establishments in Roscoff (Finistère)

Christel Douard et avec la collaboration de Valérie Guesnier

\section{Introduction}

1 Roscoff s'inscrit, depuis le troisième quart du $\mathrm{XIX}^{\mathrm{e}}$ siècle, au rang des territoires innovants en matière de recherche scientifique et de santé. Le laboratoire de biologie marine et les six établissements de santé qui s'implantent sur son littoral entre 1870 et 1930 acquièrent très tôt une renommée nationale et pour certains, internationale. Cependant, leur contribution notable à l'histoire des sciences et à la santé publique demeure aujourd'hui confidentielle en dehors de leurs propres réseaux professionnels. Les travaux de recherche historique et le recensement du patrimoine immobilier, mobilier et technique menés depuis 2010 par l'association HeSCO (Héritage scientifique et culturel Ouest) tendent à sensibiliser le public à la richesse de cette aventure scientifique et médicale qui a forgé l'identité du territoire. Les actions menées ont récemment permis la sauvegarde et l'amorce d'un programme de valorisation portant sur des éléments patrimoniaux majeurs situés dans les sanatoriums marins de Perharidy et du Laber.

\section{Soigner sur le littoral breton}

2 Le terme de sanatorium est définitivement adopté en France après le congrès national de la tuberculose qui se tient à Paris en 1893, dix ans après la découverte du bacille responsable de cette maladie infectieuse par le médecin allemand Robert Koch. Les 
institutions thérapeutiques construites alors témoignent du dramatique problème de santé publique que représente ce fléau qui touche l'Europe entière. Pourtant, la France accuse quelque retard par rapport à d'autres pays comme l'Allemagne, la Suisse ou les États-Unis, à l'exception toutefois des établissements maritimes dont l'un des plus importants dans le traitement des enfants atteints de tuberculose osseuse est présenté ici, le sanatorium de Roscoff (fig. 1).

Figure 1

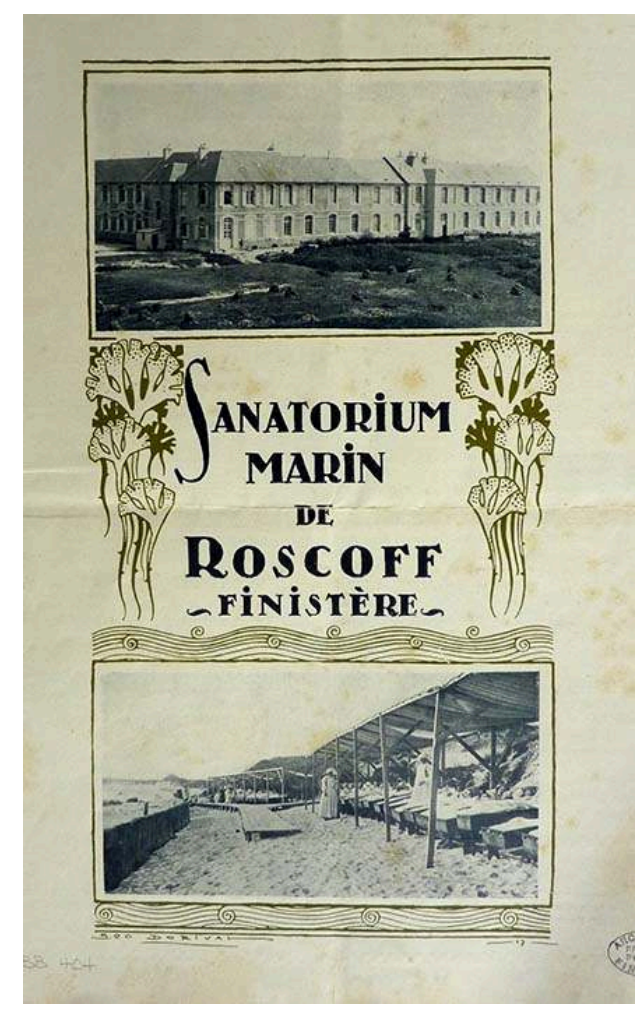

PAgE DE COUVERTURE DE LA BROCHURE DE PRÉSENTATION DU SANATORIUM MARIN DE ROSCOFF, 1917. ARCHIVES DÉPARTEMENTALES dU FINISTÈRE, Q4 BB 404.

Repro. Douard, Christel. (C) Association HeSCO, Saint-Pol-de-Léon.

Les bienfaits, supposés ou réels, de séjours prolongés sur le littoral, au contact de l'eau de mer et du soleil, avaient été mis en avant dès 1752 par le médecin anglais Richard Russel et avaient inspiré les fondateurs de l'un des plus anciens hôpitaux maritimes civils, le Royal Sea Bathing Hospital à Margate (Kent), construit en 1791 et très tôt destiné au traitement d'enfants atteints de tuberculose ${ }^{1}$.

En 1905, le littoral français compte une quinzaine d'établissements appelés hôpitaux marins, hôpitaux maritimes, sanatoriums marins, sanatoriums maritimes ou encore sanatoriums héliomarins, tous spécialisés dans le traitement de la tuberculose, principalement osseuse. Il en existe alors deux en Bretagne: Pen Bron à La Turballe (Loire-Atlantique) et Perharidy à Roscoff, entièrement réservés aux enfants ${ }^{2}$.

5 Durant le premier quart du $\mathrm{xx}^{\mathrm{e}}$ siècle, la tuberculose est responsable d'un tiers de la mortalité totale dans le Finistère. Les écoliers du primaire, estimés à environ 100000 , sont particulièrement touchés. Or, avant 1914, seuls les pavillons d'isolement de l'hôpital maritime militaire de Brest et Perharidy dispensent des traitements adaptés ${ }^{3}$ (fig. 2). 


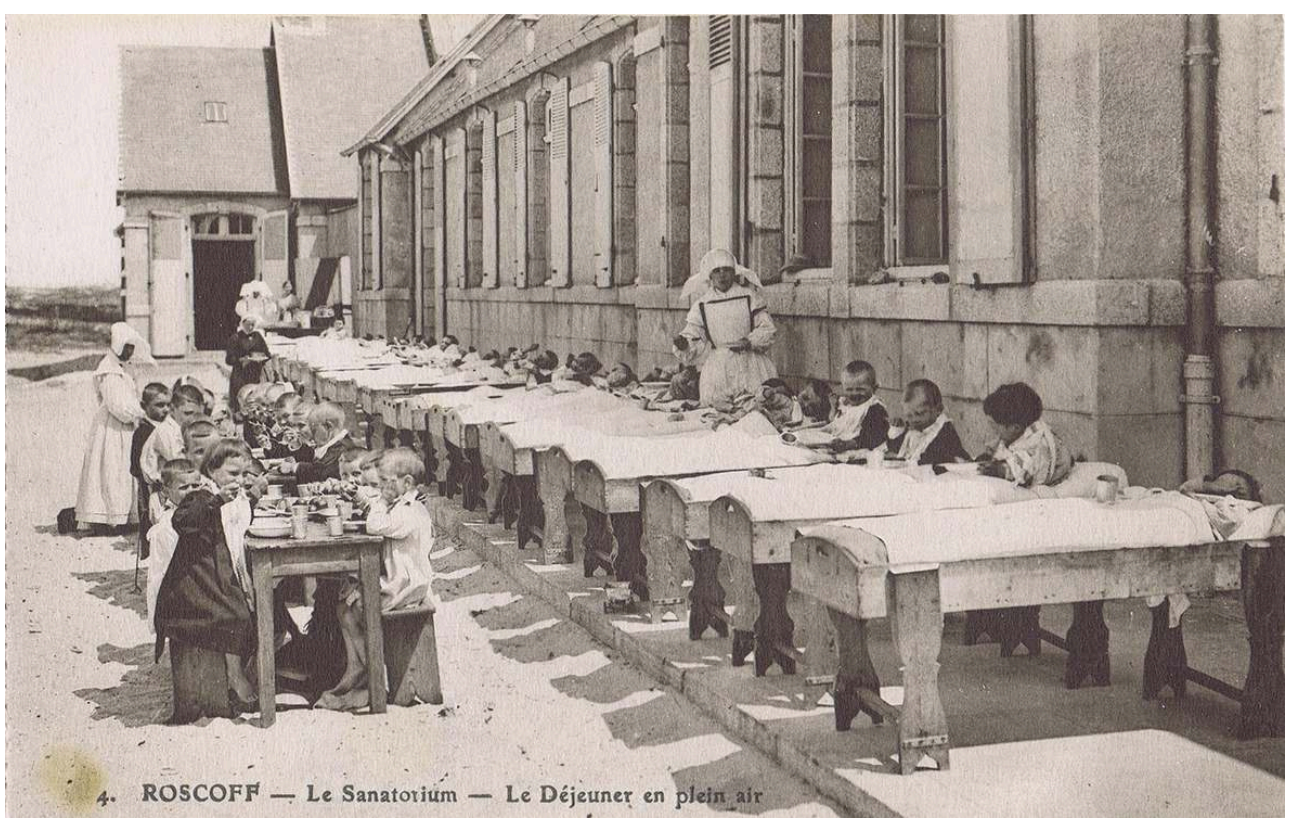

LE dÉJeUner en PLEIN AIR. CARTE POSTALE, Vers 1910.

(C) Collection Anquet, Roscoff.

6 On tentera de retracer la genèse et l'évolution du sanatorium de Roscoff, aussi bien dans son environnement naturel qu'historique, avant de mettre l'accent sur les commanditaires, les architectes et les décorateurs intervenus activement durant plusieurs décennies.

\section{Roscoff la maritime}

7 Avant de devenir un lieu de recherche scientifique et de thermalisme marin, Roscoff fut, dès le $\mathrm{XvI}^{\mathrm{e}}$ siècle, un port marchand actif ouvert sur l'Europe; son commerce maritime florissant lui a apporté une richesse dont témoigne aujourd'hui encore son remarquable patrimoine religieux et civil.

8 À la fin du XIX siècle, cet héritage historique attire les touristes séduits par la vieille ville et le pittoresque du front de mer pendant que les premiers patients commencent à fréquenter les établissements de santé. La liaison ferroviaire Paris-Roscoff établie en 1883 permet à cette clientèle, mais également à une communauté scientifique internationale d'accéder directement à son lieu de séjour.

9 Scientifiques et médecins découvrent, à la même époque, un site exceptionnel tant par sa biodiversité marine et la richesse de son champ d'algues que par la qualité de son climat particulièrement tempéré, tonique et stimulant. Ils fondent alors deux établissements devenus emblématiques de la recherche scientifique et de la santé, la station biologique et l'institut marin de Roc'h Roum.

Lieu voué à la science, la station biologique doit son existence et sa renommée à Henri de Lacaze-Duthiers, professeur de zoologie à la Sorbonne et membre de l'Académie des sciences. Il fonde le laboratoire en 1872, dix ans avant celui de Banyuls-sur-Mer (Pyrénées-Orientales) destiné, en Méditerranée, au même type de recherches; comme à 
Roscoff, un grand sanatorium marin pour enfants y fonctionne dès $1883^{4}$. Désireux de rendre à la science française son niveau d'excellence et d'élever la zoologie au rang des sciences expérimentales, Lacaze-Duthiers innove en privilégiant les études en milieu naturel. Les chercheurs du monde entier vont y trouver et y trouvent encore des conditions de travail idéales ${ }^{5}$.

11 L'institut marin de Roc'h Roum est créé en 1902 par le médecin Louis Bagot ${ }^{6}$, convaincu des bienfaits du microclimat local associé aux vertus thérapeutiques de l'eau de mer chauffée et des algues. En traitant les rhumatismes par hydrothérapie il pose, le premier, les bases de la thalassothérapie moderne. Il sera également le second médecin du sanatorium de Perharidy.

12 C'est dans cet environnement favorable à la recherche et à la santé que naît le sanatorium marin de Perharidy dont les liens avec l'institut marin sont particulièrement étroits dès l'origine des deux établissements (fig. 3).

Figure 3

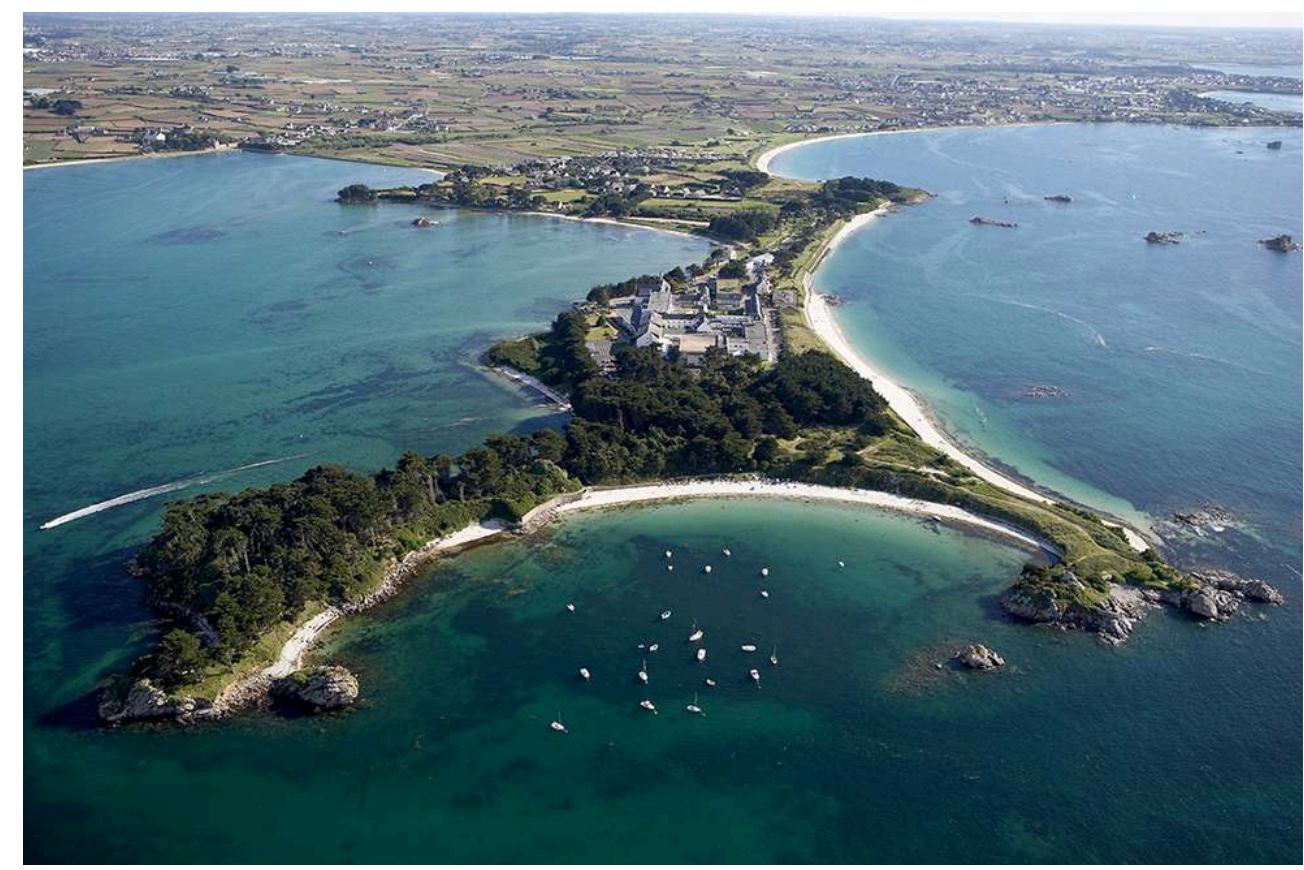

vUe AÉrienne ACtuelle de LA PResqu'île de PERHARIdy, dePUIS LE NORd. AU CENTRE, L'ÉTABLISSEMENT, À gAUCHE, L'ANSE DU LABER.

(C) FONDATION ILDYS/CENTRE DE PERHARIDY.

\section{Perharidy : un sanatorium héliomarin}

\section{Une initiative privée exemplaire}

13 Louise de Kergariou (1854-1915) avait épousé Charles-Marie de Kergariou, grand propriétaire terrien et député des Côtes-du-Nord. Sans descendance et animée de charité chrétienne, elle décide, après la mort de son mari en 1897 , de consacrer sa fortune et son énergie à la création d'un établissement pour enfants atteints de tuberculose. Elle prospecte d'abord le littoral voisin du Trégor, notamment les environs de Saint-Michel-en-Grève, avant d'acquérir en 1900 une presqu'île inhabitée d'environ 
trois hectares située entre Roscoff et l'île de Batz, un bout de terre sablonneux, de faible hauteur et dépourvu de végétation ${ }^{7}$. Seule l'extrémité nord est occupée par un fort désaffecté qui avait été érigé en 1861 en tant que maillon de défense de la Manche.

Louis Bagot, médecin et directeur de l'institut marin, et Albert Calmette, célèbre bactériologiste, fondateur du premier dispensaire antituberculeux en France (Lille, 1901-1903), surtout connu pour sa mise au point du vaccin contre la tuberculose, s'expriment en faveur du choix du site. Ce dernier suit d'ailleurs durant trente ans l'évolution de l'établissement; il se rend à plusieurs reprises à Roscoff et en 1928, il souligne son exemplarité au niveau national ${ }^{8}$.

La fondation est reconnue d'utilité publique dès 1902, un an après la sortie de terre - ou plutôt de sable - du premier bâtiment (fig. 4) pour lequel, en l'absence de voie d'accès, on fait transporter les matériaux de construction par la grève, à marée basse. La congrégation des Sœurs de la Charité de Saint-Vincent-de-Paul et celle des Sœurs de la Présentation assurent le fonctionnement de l'établissement jusqu'en 1932, avant de se consacrer exclusivement aux soins et à l'intendance.

Figure 4

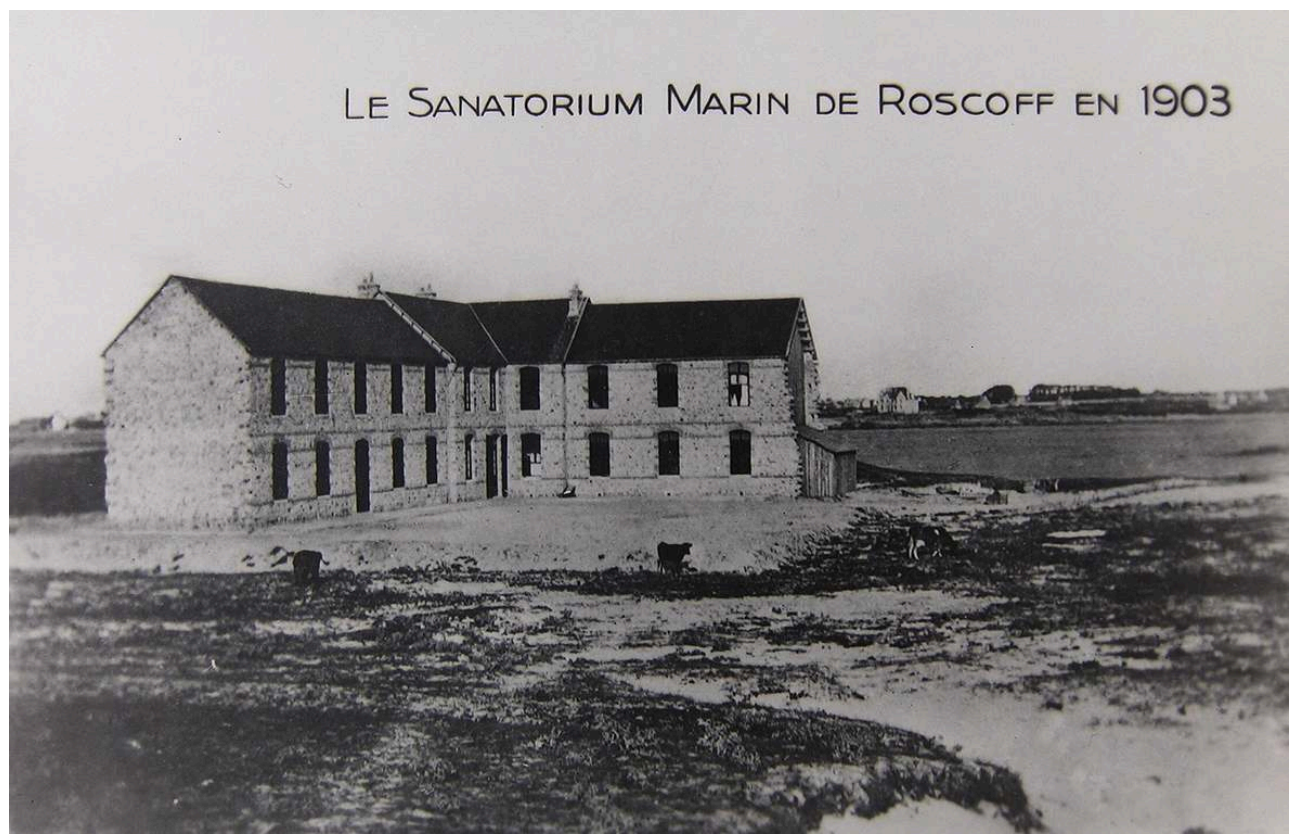

LE SANATORIUM MARIN DE ROSCOFF EN 1903. CARTE POSTALE.

(C) Association HeSCO, Saint-Pol-de-Léon.

Le projet architectural, relativement ambitieux, ne sera mis en œuvre que progressivement, comme le précise Louise de Kergariou qui prévoit d'emblée « un vaste hôpital à construire par étape, avec des constructions simples formant deux rectangles, cours protégées du nord et de l'est et d'ouest par des bâtiments à étages, aile sans étage au $\operatorname{sud}^{9}$ ». Résidant sur place, elle occupe un petit appartement associé à une chapelle domestique - disparue mais connue par une photographie ancienne - dans le premier bâtiment achevé.

Après la mort de Louise de Kergariou, en 1915, sa nièce Hélène de Fontenilliat continue l'œuvre philanthropique entreprise ; elle préside la fondation jusqu'à son propre décès en 1958, époque où la tuberculose est pratiquement éradiquée. C'est elle, aidée par une 
équipe dévouée, qui, en investissant les fonds propres de la fondation et en obtenant des subventions publiques, continue à compléter, à moderniser et à agrémenter l'établissement. Après le rapprochement avec le site voisin du Laber en $1920^{10}$, lui aussi voué aux soins antituberculeux, la réputation du sanatorium, dont la capacité d'accueil est alors sensiblement augmentée, rayonne bien au-delà du cadre local et régional.

\section{Une presqu'île pour les enfants}

Si le site paraît, aujourd'hui encore, sauvage, enchanteur et pittoresque, il pouvait être ressenti par les petits malades comme un lieu synonyme de tristesse et d'isolement que les aménagements successifs ont toutefois amélioré et rendu aussi agréable que possible.

Avant la mise en place d'une route terrestre en 1917, il fallait traverser la grève du Laber à marée basse pour rejoindre l'établissement. Le caractère contagieux de la maladie, mis en évidence dès 1865 par Jean-Antoine Villemin, plaidait pour cet isolement préconisé par les médecins.

Le concept de "plein air » reprend celui de «wilderness » né aux États-Unis ${ }^{11}$ dans la seconde moitié du xIX ${ }^{e}$ siècle, selon lequel l'environnement est un facteur déterminant de l'apparition, de la propagation, mais aussi de la guérison de la tuberculose. La cure marine, la lumière, l'air iodé vivifiant et les bains de mer seront longtemps considérés comme bénéfiques pour soigner les enfants atteints de tuberculose osseuse et ganglionnaire, de rachitisme et d'anémie (fig. 5). Louise de Kergariou, impressionnée par la création du sanatorium pour enfants à Berck (Pas-de-Calais) et touchée par l'indigence d'une partie de la population finistérienne, fait donc aménager ce lieu sauvage parfaitement adapté, où les températures moyennes, considérées comme idéales, oscillent entre $17^{\circ} \mathrm{C}$ en été et $8^{\circ} \mathrm{C}$ en hiver ${ }^{12}$. 


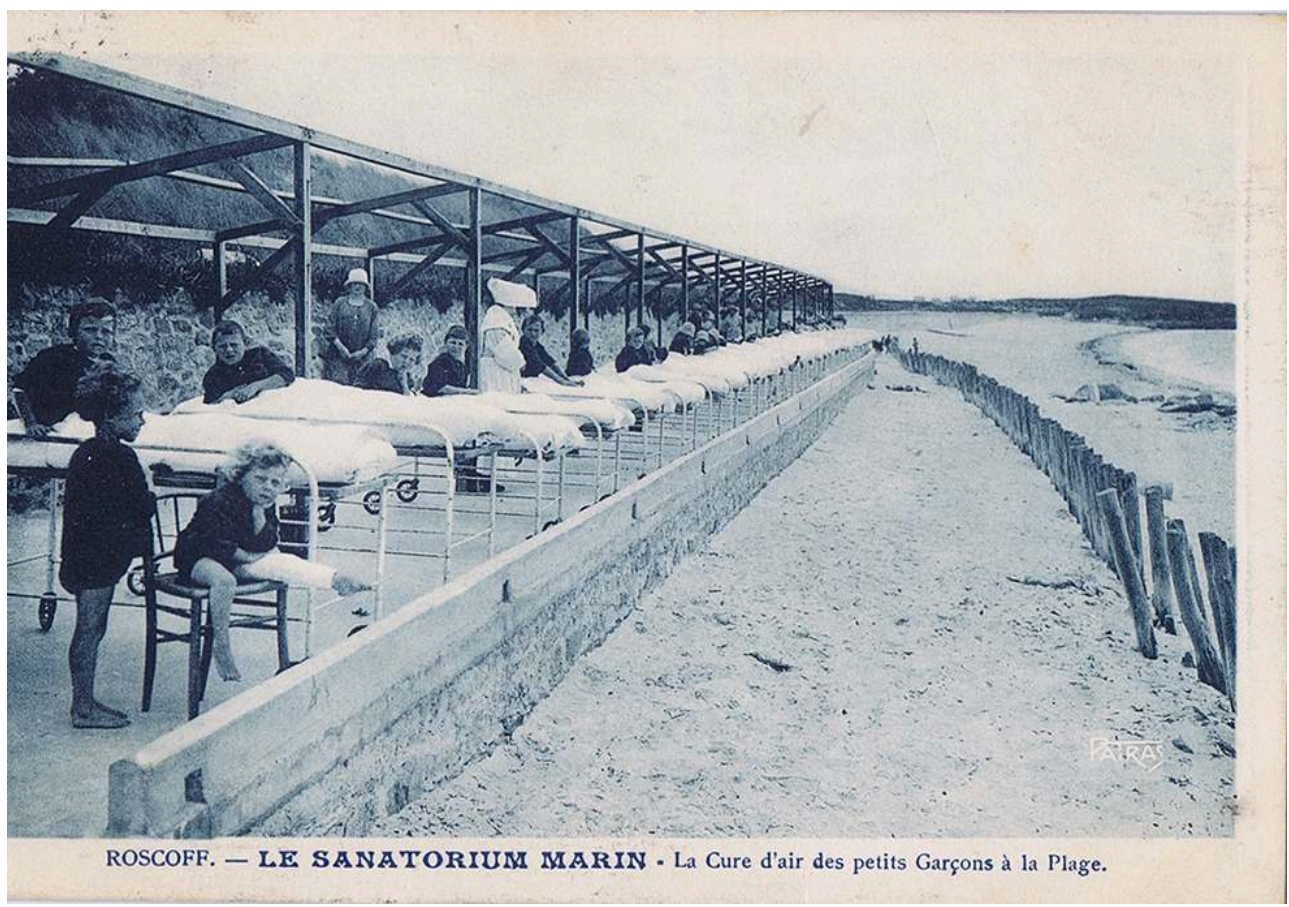

LA partie sud de la cure d'air au début des anNées 1930. CARTe postale.

(C) Association HeSCO, Saint-Pol-de-Léon.

Dès 1901, l'établissement accueille des filles de 3 à 21 ans et des garçons de 3 à 14 ans de toute la France, rarement des environs proches ou du département. À peu près 40 lits sont répartis dans deux grands dortoirs avec, au centre, une cuisine, une chapelle, une salle de chirurgie ainsi que, comme le souligne la fondatrice, un « pavillon copié sur les pavillons d'isolement et box vitrés de l'hôpital Pasteur, aménagé suivant les indications du professeur Calmette $»^{13}$.

Des communs - logement du jardinier, buanderie, écurie, basse-cour et porcherie - sont adjoints progressivement. Un jardin potager permet une vie en quasi-autarcie alimentaire, une nourriture saine et abondante étant considérée comme un élément primordial du traitement. Deux puits - le premier était en 1904 actionné par un manège à cheval - assurent la distribution en eau potable. Des cyprès Lambert et des ficoïdes sont plantés sur des terrains encore dénudés afin d'éviter l'éboulement des dunes, surtout celles très exposées à l'ouest.

Les ravages de la Première Guerre mondiale et l'inquiétante progression de la tuberculose confortent plus que jamais la direction de Perharidy dans son combat contre la maladie et son projet d'agrandissement afin de «soigner les enfants qui seront la France de demain ${ }^{14} »$. L'établissement reçoit le soutien financier et moral de diverses organisations. La Croix-Rouge américaine, l'organisation britannique «Save the Children " ainsi que des fondations privées à caractère caritatif telles que Rockefeller, Cognacq-Jay et Post, s'avèrent particulièrement généreuses. Ces aides substantielles débloquées pour la lutte antituberculeuse, en pleine guerre contre l'Allemagne, reposent, bien évidemment, sur des motivations sanitaires et philanthropiques, mais aussi politiques. Ainsi, Henri Lavedan, membre de l'Académie française, écrit en 1917 dans la préface de la première brochure présentant le sanatorium héliomarin de Roscoff : 
Après avoir purgé le sol de l'ennemi qui l'occupe et l'infeste, il faut libérer l'autre sol, l'autre territoire, celui de la santé française, de l'autre ennemi qui l'envahit et la ravage. (...) Tous ces petits que, grâce à la pieuse tendresse de votre personnel et avec le secours divin du soleil, de la brise marine et des célestes baumes, vous rendez à la vie, sont des enfants deux fois ressuscités, surtout quand ils ont perdu leur père ${ }^{15}$. devant le conseil général, le Dr Lucas, parlant du sanatorium de Perharidy, déclare que «les résultats sont vraiment extraordinaires. Des enfants qui ont été apportés mourants en ressortent trois mois après gambadant et courant. Il y a intérêt à sauver l'enfant avant de sauver l'homme ${ }^{17}$. Les pouvoirs publics apportent alors des financements significatifs : grâce au contrat liant le conseil général à la fondation de Perharidy, les travaux d'agrandissement peuvent être engagés rapidement, à condition de réserver 70 lits aux enfants pauvres originaires du département (fig. 6).

Figure 6

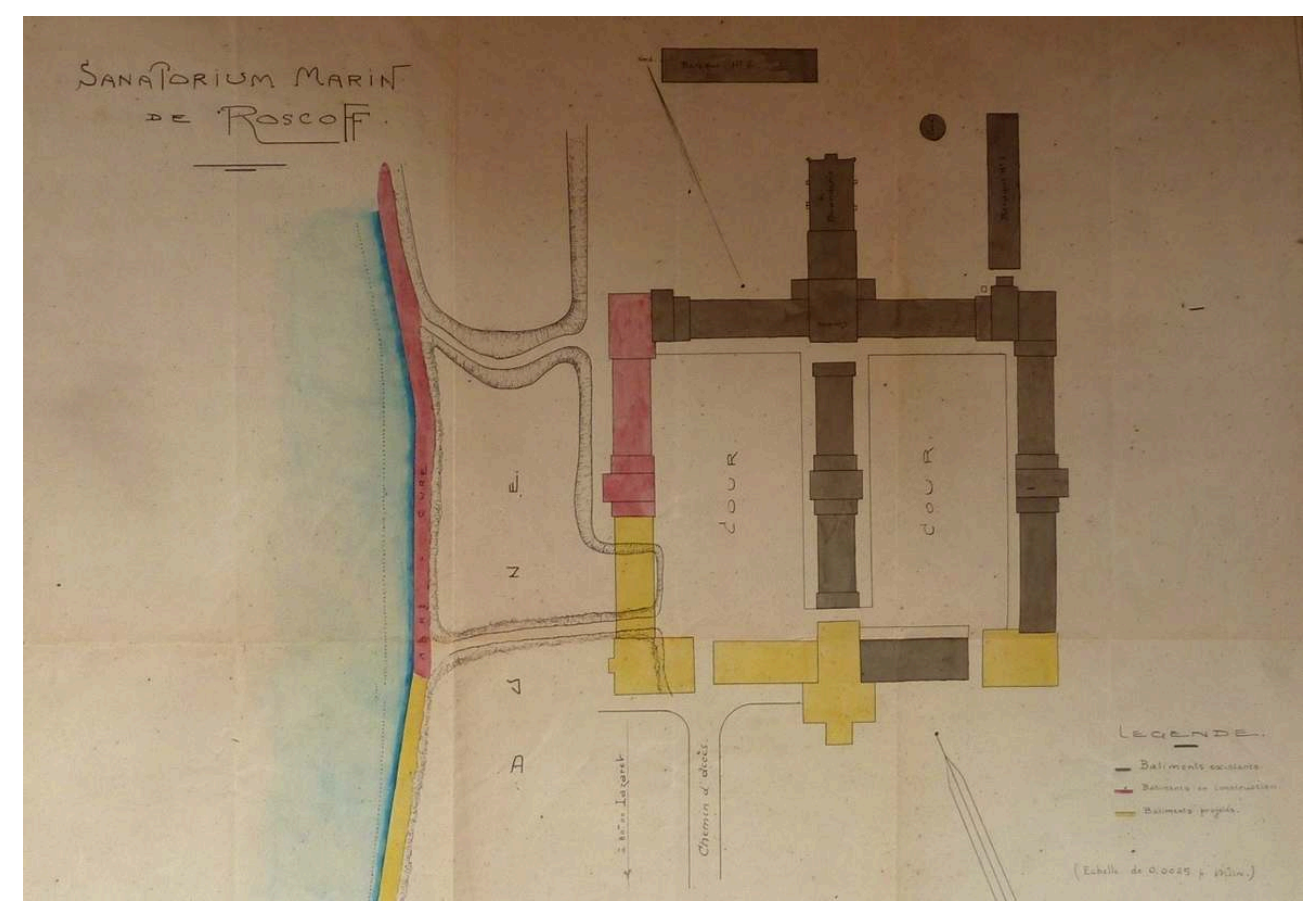

PLAN DU SANATORIUM EN 1919. EN GRIS : BÂTIMENTS CONSTRUITS ; EN ROUgE : TRAVAUX EN COURS ; EN JAUNE : BÂTIMENTS PROJETÉS. ARCHIVES DÉPARTEMENTALES DU FINISTÈRE, 1 X 149.

Repro. Douard, Christel. @ Association HeSCO, Saint-Pol-de-Léon.

Les documents figurés de cette époque montrent les enfants valides faisant de la plage ou des cours du sanatorium leur terrain de jeu; d'autres sont alités sur des brancards posés à même la grève ou sous les auvents aménagés le long des bâtiments, dans un univers de sable, de soleil et de vent.

27 Malgré l'omniprésence de la maladie, perceptible au travers de plâtres ou d'appareillages dont sont pourvus certains enfants, les photographies donnent une image plutôt positive des lieux. De même, un document cinématographique étonnant : 
en 1917, le gouvernement français avait demandé à Jean Comandon (1877-1970), médecin et précurseur du cinéma scientifique, de réaliser une série de films pour la campagne de propagande antituberculeuse lancée par la Croix-Rouge américaine et la fondation Rockefeller. Une séquence de 15 minutes est tournée un an plus tard à Perharidy ${ }^{18}$. Autorités politiques et médicales, dont Léon Bourgeois, président du Conseil, et Léon Bernard, professeur de médecine, se déplacent pour soutenir les actions de lutte contre la tuberculose dans un sanatorium héliomarin pour enfants considéré comme exemplaire et à la pointe du progrès. Devant ce succès, une nouvelle phase d'agrandissement et de modernisation est lancée, portant la capacité d'accueil de l'établissement jusqu'à 600 enfants malades en 1934.

\section{Santé et architecture entre 1901 et $1938^{19}$}

28 Autour de 1900, les architectes sont peu préparés à ces chantiers d'un type relativement nouveau. Il n'est pas rare que les médecins interviennent dans les choix architecturaux pour imposer des cahiers des charges essentiellement fonctionnalistes.

À Perharidy, l'architecte Joseph-Charles de Montarnal ${ }^{20}$ opte pour des bâtiments à étages fort simples et des façades sévères et ordonnancées qui se distinguent peu de certains immeubles urbains. Montarnal avait pourtant fait un choix bien différent pour le fameux institut orthopédique de Berck-sur-Mer (Pas-de-Calais), contemporain de Perharidy. Il propose aussi le projet pour un établissement de type hôtelier, la Maison Saint-Luc - une version réduite de Berck - réalisée à la même époque à Roscoff, en face de Perharidy ${ }^{21}$. Il est difficile de savoir dans quelle mesure Louis Bagot, médecin attitré à Perharidy dès 1902, a pu peser sur les choix architecturaux. L'aspect extérieur des bâtiments lui importait sans doute moins que les aménagements intérieurs, qui devaient répondre aux exigences médicales.

Les documents originaux du projet de Montarnal n'ont pas été retrouvés à ce jour mais sa proposition en forme de quadrilatère a visiblement été reprise en 1917 par l'architecte Laurent ${ }^{22}$. Dès 1918, les pavillons destinés à l'isolement, à la chirurgie, à la radiographie et à l'orthopédie sont opérationnels. L'achèvement de l'ensemble des bâtiments, qui connaîtront des transformations, semble se situer autour de 1930 (fig. 7, fig. 8). Les mesures d'hygiène appliquées dans tout l'établissement sont draconiennes : Albert Calmette se déplace en personne en 1920 pour vérifier que «l'eau, les fosses, la stérilisation et l'alimentation sont d'une qualité irréprochable ${ }^{23} »$. 
Figure 7

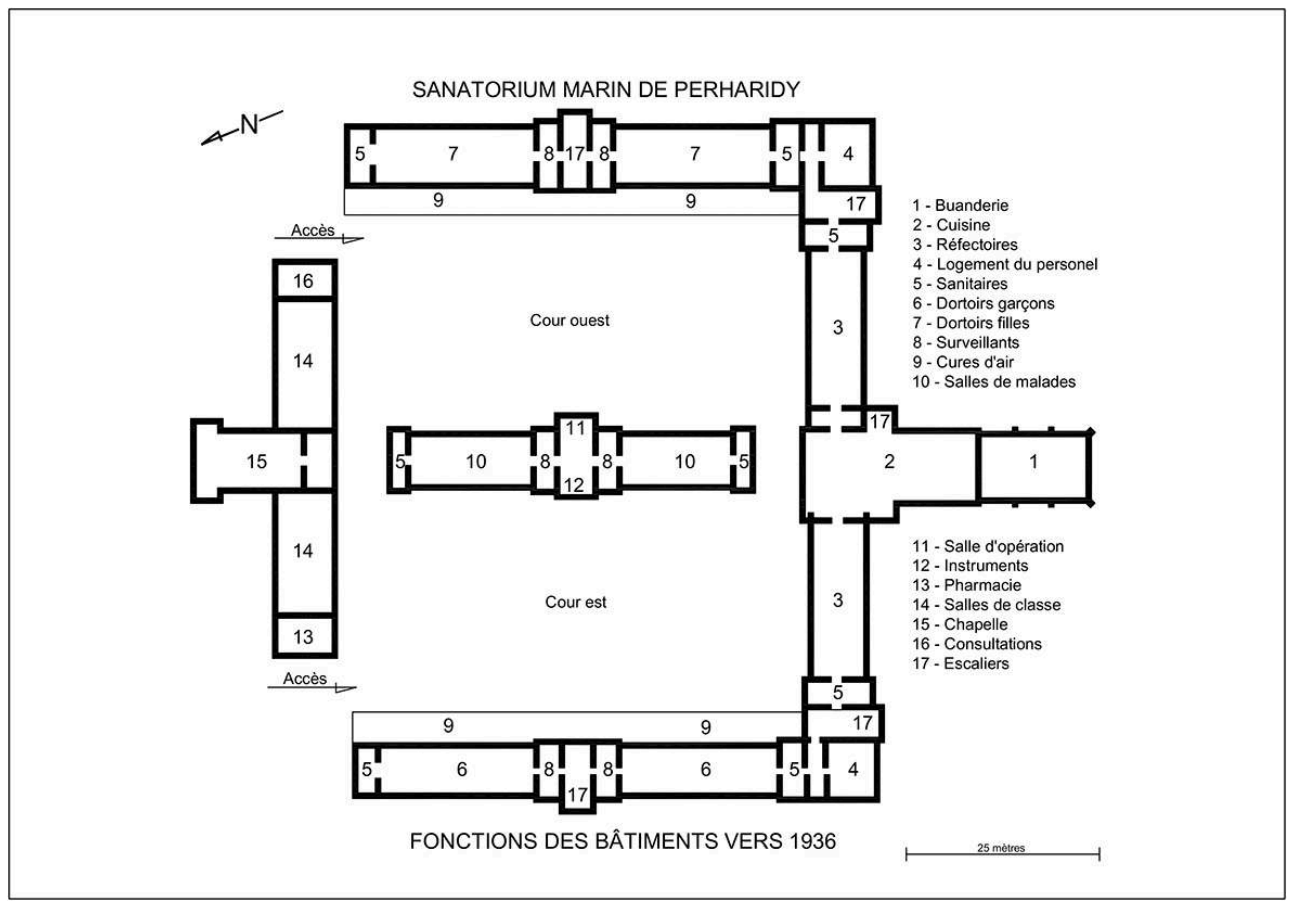

FONCTIONS SUPPOSÉES DES BÂTIMENTS VERS 1930. PLAN BERNARd VAVASSEUR (À PARTIR D'UN DOCUMENT DE 1917).

(c) Association HeSCO, Saint-Pol-de-Léon.

Figure 8

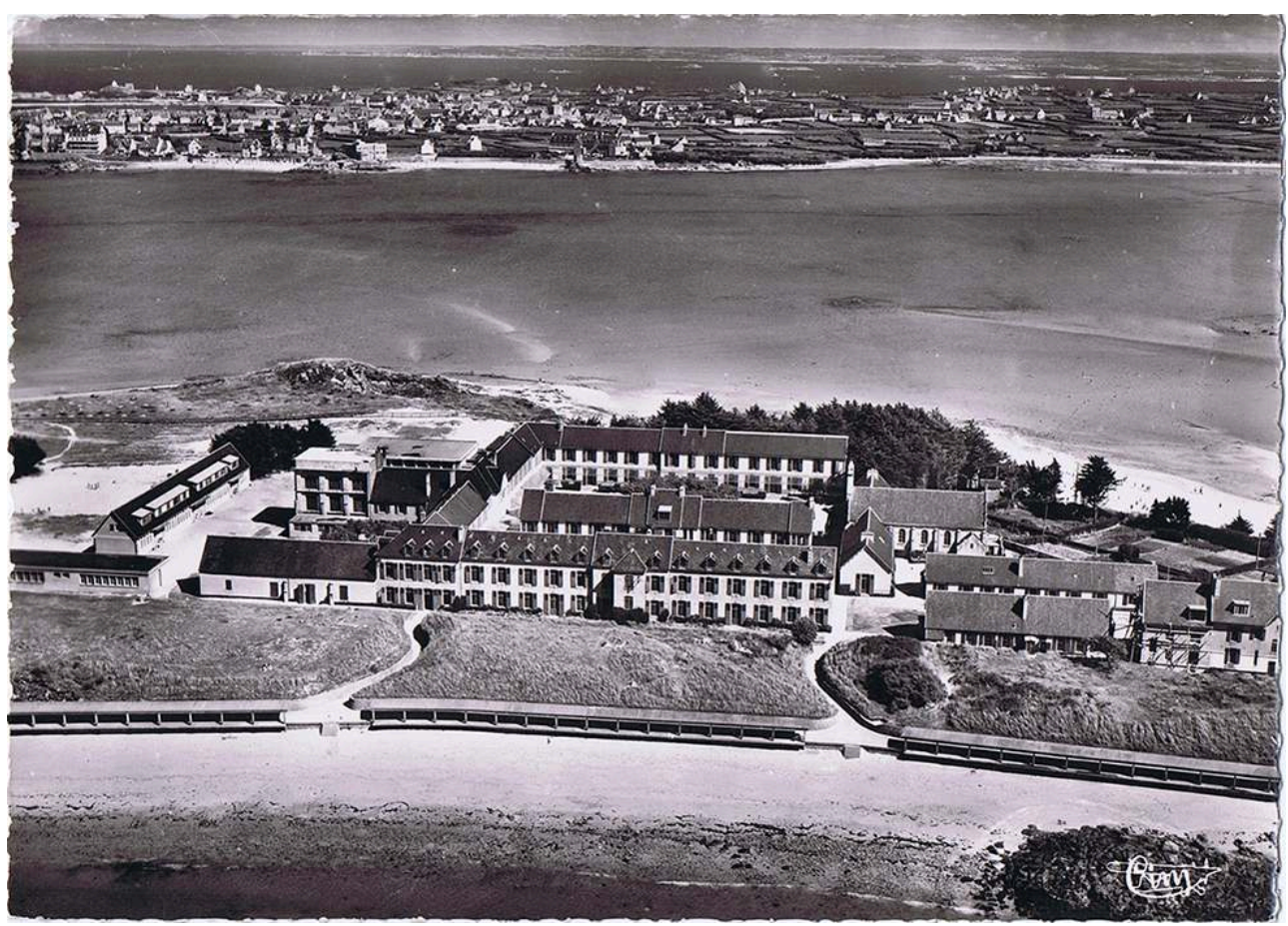

VUE AÉRIENNE, VERS 1935. ON DISTINgUe LeS digUes ASSOCIÉES À LA CURE d'AIR, LA BAIE dU LABER ET ROSCOFF ET, AU FOND, LA BAIE DE MORLAIX. À DROITE, L'ANCIENNE MAISON DU DIRECTEUR.

(c) Association HeSCO, Saint-Pol-de-Léon. 
Après la Première Guerre mondiale, les autorités se mobilisent pour endiguer « la peste blanche ", notamment grâce à la loi Honorat de 1919 qui impose l'installation d'un sanatorium ou d'un préventorium dans chaque département. C'est une époque charnière dans la conception de ces lieux de soins. L'architecture s'éloigne des formes héritées de la seconde moitié du xix siècle et les architectes, face aux nouveaux programmes, commencent à proposer des solutions originales et novatrices.

Cette modernité n'est pas sensible à Perharidy, où le projet initial né au début du siècle n'est pas remis en cause. On continue à agrandir et à compléter, toujours suivant le plan d'origine de Montarnal, tout en remplaçant à plusieurs reprises les constructions secondaires à usage technique et en rajoutant, par exemple, plusieurs salles de classe pour un nombre croissant d'enfants scolarisés. En 1936, on compte six classes, pouvant accueillir 200 écoliers valides ${ }^{24}$.

Composé de quatre ailes ouvertes sur deux cours accessibles uniquement par deux passages étroits au sud, le sanatorium de Perharidy répond encore à cette structure classique qui s'inscrit dans la longue tradition des hôtels-Dieu alors que les plans pavillonnaires ou « en peigne » étaient répandus dans l'architecture hospitalière depuis le milieu du XIX ${ }^{e}$ siècle $^{25}$. Les cuisines (nourriture des corps) et la chapelle (nourriture des âmes) se font encore face. Comme dans l'architecture carcérale, la surveillance doit être possible d'un coin à l'autre de cet espace fermé, tout en facilitant les circulations entre les différentes parties de l'ensemble. À l'exception de l'aile sud (anciennes salles de classe, chapelle), les pavillons à étages abritant les dortoirs sont globalement identiques.

La séparation des sexes est respectée : la partie est (ailes et cour) est réservée aux garçons, la partie ouest aux filles. Les préaux, couverts de toits en verre, établis le long des rez-de-chaussée, servent non seulement à abriter les lits pour la cure d'air mais facilitent aussi la circulation en cas de mauvais temps. Cuisine (fig. 9) et office occupent la partie centrale de l'aile nord alors que le pavillon central situé au centre de la cour abrite les parties réservées aux soins médicaux (salle de soins, salle d'opération, pharmacie...). 
Figure 9

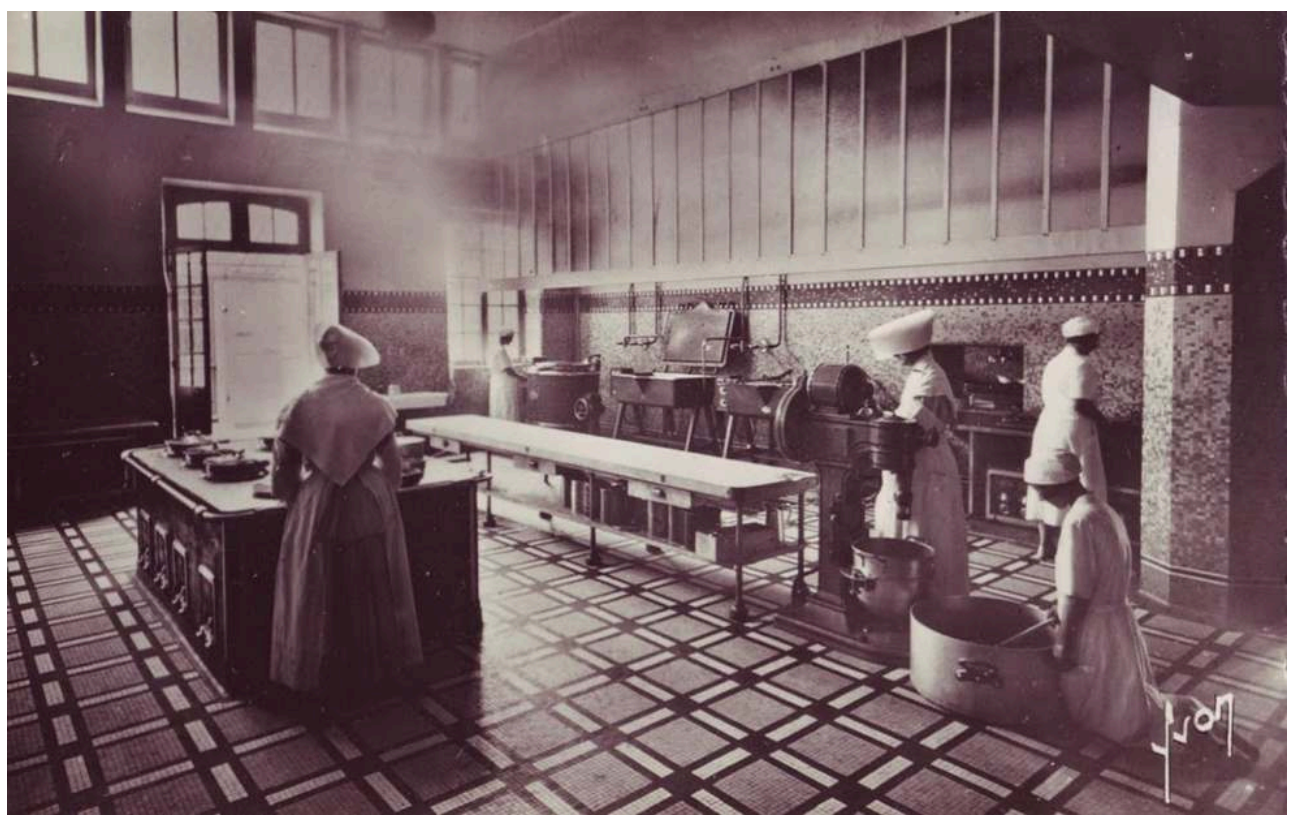

LA NOUVELLE CUISINE VERS 1936. DES MOSAÏQUES D'OdORICO COUVRENT SOL, LAMBRIS ET PILIERS. CARTE POSTALE.

(c) Association HeSCO, Saint-Pol-de-Léon.

Une certaine rupture dans le parti architectural, somme toute modeste, ne se fait sentir qu'à travers un seul bâtiment, la chapelle, certes prévue dès l'origine mais dont la réalisation n'intervient qu'en $1922^{26}$. CEuvre de l'architecte Lionel Heuzé, de Morlaix, elle tranche stylistiquement avec les éléments construits vingt ans plus tôt. Liée aux familles bienfaitrices de l'établissement durant plusieurs décennies, elle suit un plan allongé en T sur lequel se greffe une chapelle funéraire au sud, ajoutée en 1934 (fig. 10). La charpente est un mélange de composantes stylistiques issues du Moyen Âge anglais et breton. Ces éléments sont typiques des réalisations de l'architecte, adepte du néorégionalisme ${ }^{27}$. Par ailleurs, la modernité architecturale se manifeste également à travers deux éléments situés hors du quadrilatère : la cure d'air et le logement de fonction bâti vers 1932 pour Marcel Rousseau, directeur du sanatorium entre 1932 et 1963 et lui-même architecte $^{28}$. Appréciant le béton armé et les revêtements en mosaïque, il est à l'origine d'un grand nombre d'évolutions architecturales et d'équipements intérieurs. 
Figure 10

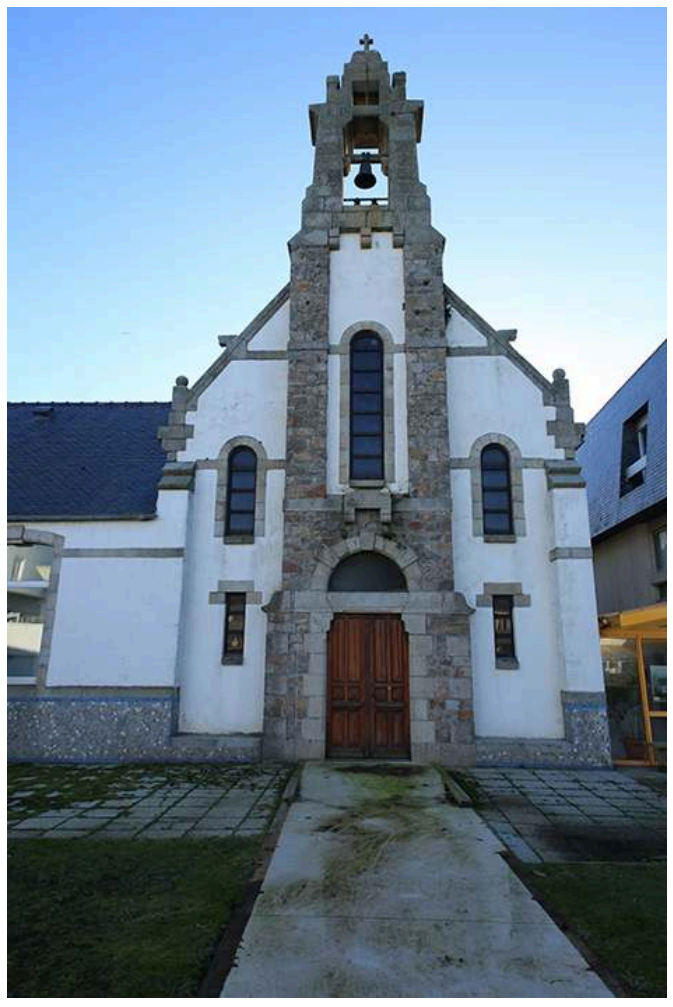

ÉLÉVATION NORD dE LA CHAPELLE CONSTRUITE EN 1922 d'APRÈS LES PLANS DE L'ARCHITECTE LIONEL HEUZÉ.

Phot. Vavasseur, Bernard. (c) Association HeSCO, Saint-Pol-de-Léon.

Aménagée dès 1911 à même la plage, la cure d'air est une belle illustration d'un dispositif architectural au service de la santé. Dédiée à l'héliothérapie et à l'exposition des enfants à l'air iodé, elle ne consiste à l'origine qu'en de simples trottoirs en bois surmontés de pare-soleil, aménagements qui permettent toutefois aux chariots et brancards des enfants invalides d'accéder à la plage. En 1919, une tempête et un raz de marée d'équinoxe entament la dune qui protège le sanatorium où résident 350 enfants et 60 personnes de service. Des travaux d'urgence «sous peine de mettre en péril l'existence de la presqu'île de Perharidy et les établissements importants du sanatorium ${ }^{29}$ " sont entrepris et une "digue à la mer " est mise en chantier. Cette protection en béton remplit en même temps la fonction de cure d'air, en remplacement des anciens trottoirs en bois. L'étendue du dispositif atteint environ 300 mètres vers 1930. C'est probablement au milieu de cette décennie, sous l'impulsion du directeur (et architecte) Marcel Rousseau, que la galerie de cure est couverte d'un vaste toit incurvé en ciment reposant sur des piles en béton, dispositif qui disparaît après la Seconde Guerre mondiale. Dans l'architecture des sanatoriums marins, une telle structure placée à même la grève est peu commune (fig. 11). 
Figure 11

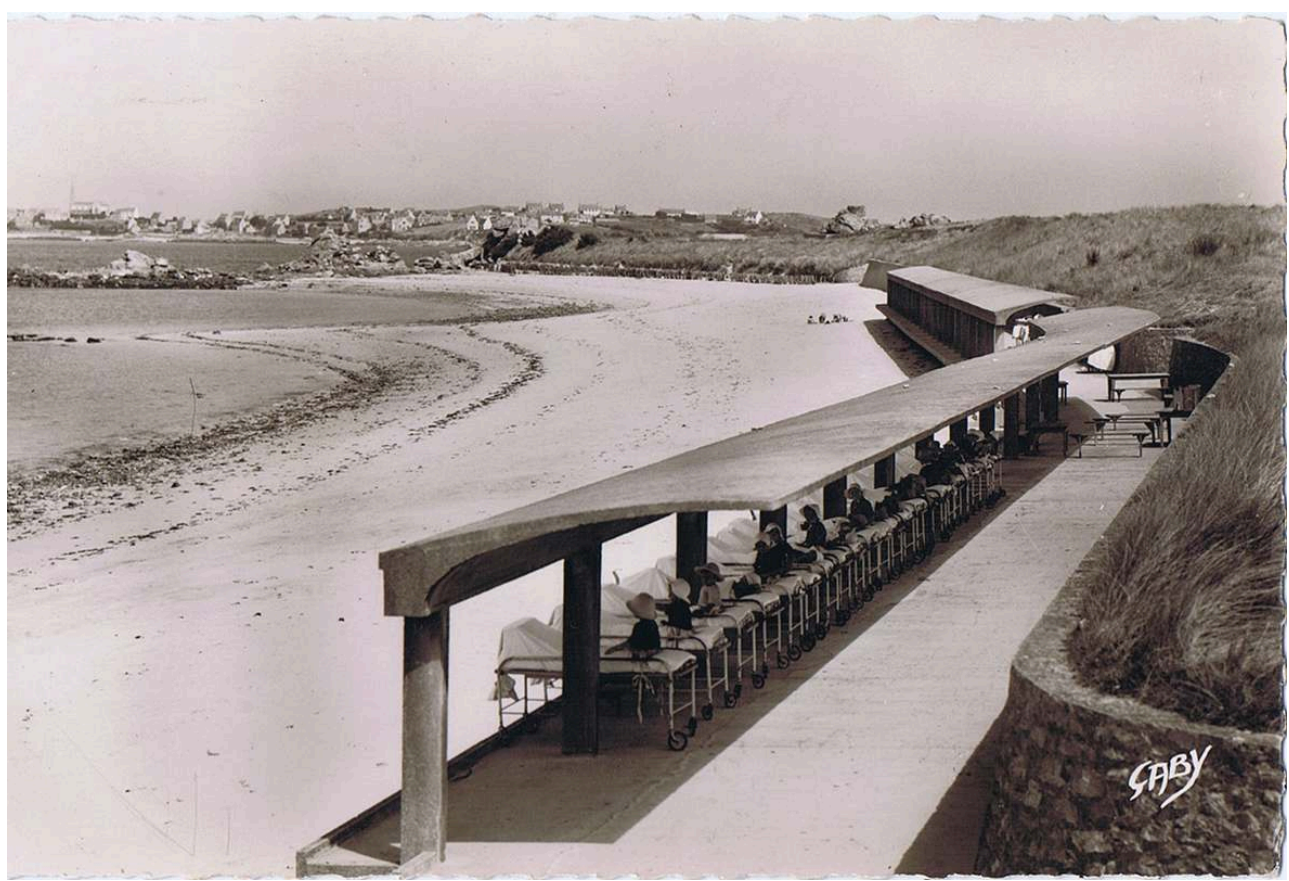

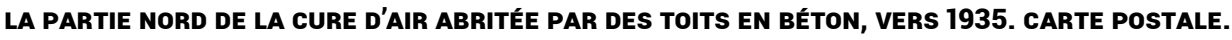

(C) Association HeSCO, Saint-Pol-de-Léon.

\section{Embellir les intérieurs}

Le sanatorium connaît son expansion maximale dans l'Entre-deux-guerres. Un soin particulier est alors porté aux aménagements intérieurs, dans un but à fois fonctionnel, hygiéniste et décoratif. En 1934, les murs de la salle des fêtes nouvellement construite reçoivent un décor peint réalisé par Charles de Kergariou dit Kerga (1899-1956) ${ }^{30}$, neveu et filleul de la fondatrice du sanatorium. Les environs de Morlaix, surtout le littoral entre Roscoff et l'île de Batz, lui fournissent une multitude de sujets évoquant le pittoresque de la Bretagne traditionnelle qu'il représente sur onze panneaux: vie portuaire, ramassage et brûlage du goémon, récolte des choux-fleurs. D’autres scènes, parmi les plus touchantes, montrent, telle une mise en abyme où l'environnement extérieur est représenté sur les parois intérieures, la vie quotidienne des enfants, les soins, les cures d'air, les jeux ou encore une partie de voile dans l'anse du Laber ${ }^{31}$ (fig. 12, 13, 14). 
Figure 12

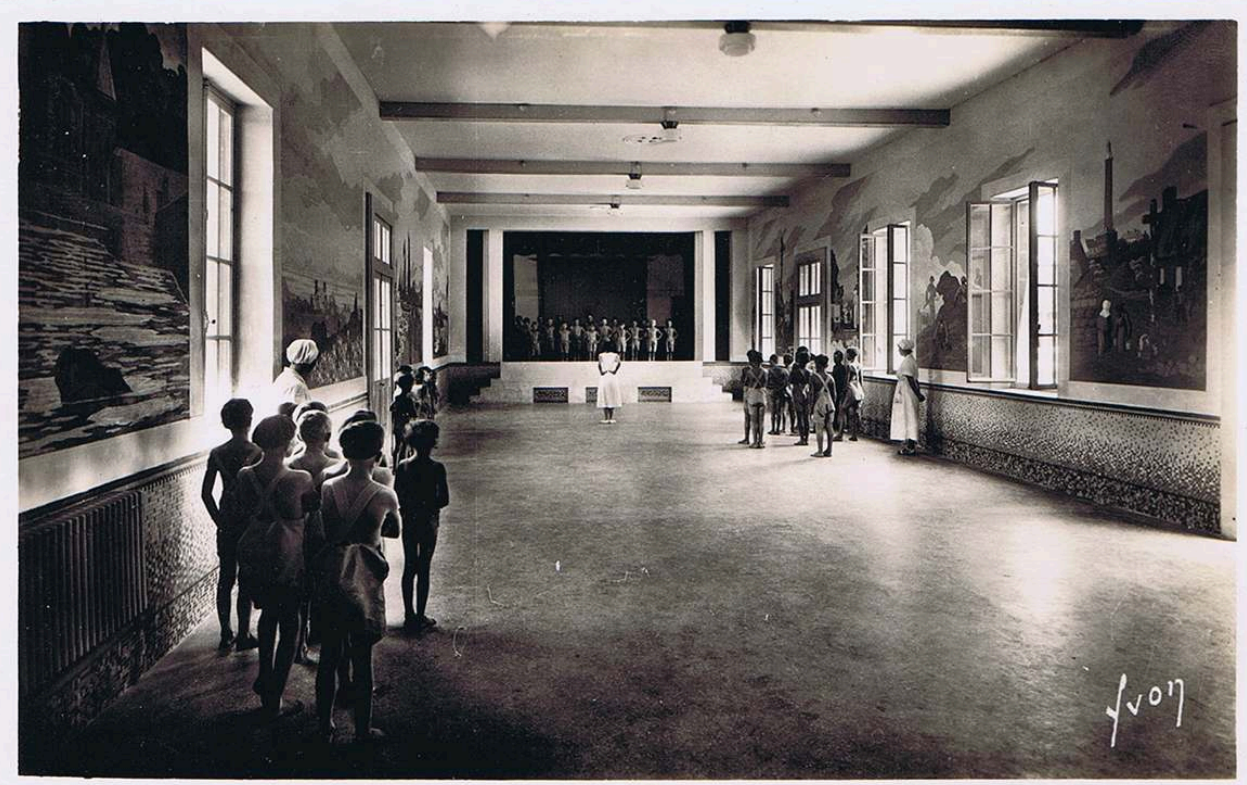

Ancienne salle des fêtes (détruite) montrant le décor mural réalisé par Charles de Kergariou, 1934. (c) Association HeSCO, Saint-Pol-de-Léon.

Figure 13

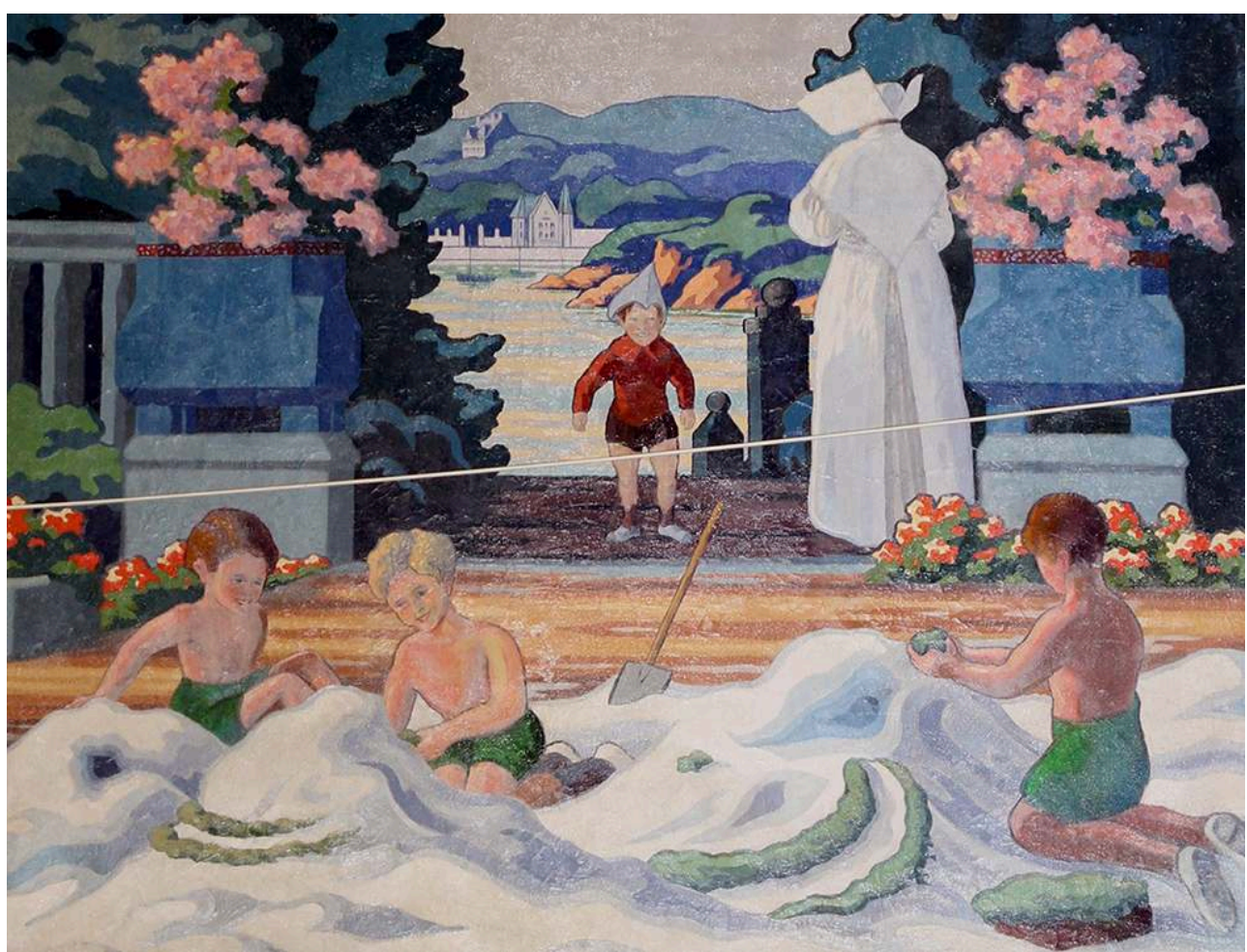

Ancienne salle des fêtes, détail d'une peinture murale réalisée en 1934 par Charles de Kergariou. Au fond, le château et la clinique du Laber.

Phot. Vavasseur, Bernard. @ Association HeSCO, Saint-Pol-de-Léon. 


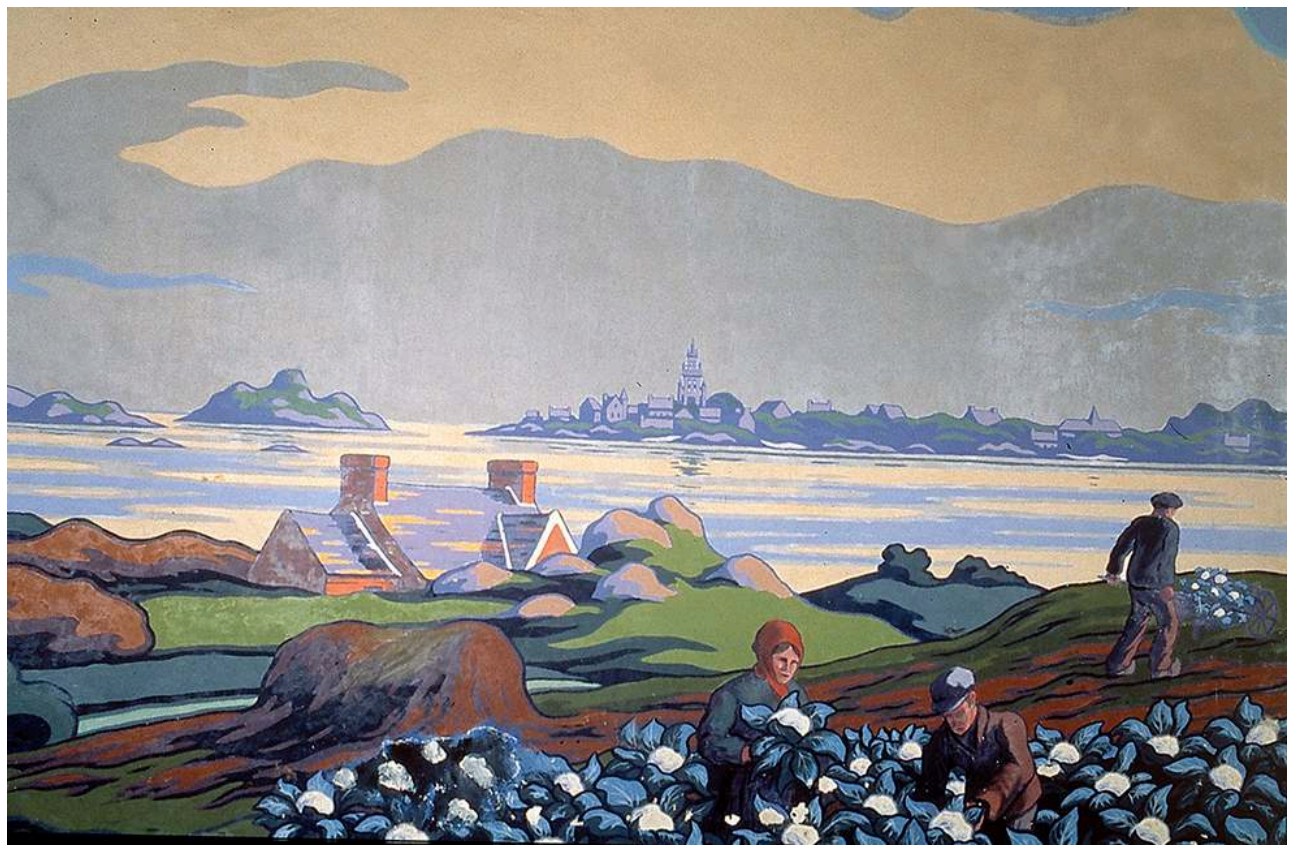

Ancienne salle des fêtes, détail d'une peinture murale réalisée en 1934 par Charles de Kergariou représentant la coupe des choux-fleurs devant la baie du Laber et la ville de Roscoff. État en 1992.

Phot. Artur, Guy. Service de l'inventaire du patrimoine culturel. (c) Région Bretagne, Rennes.

La direction de l'établissement entreprend au même moment des travaux d'une autre envergure qu'elle désigne elle-même comme « politique de la mosaïque ${ }^{32}$ ». Les salles de classe, les dortoirs, les réfectoires ou encore la cuisine et ses pièces annexes, sont couverts de mosaïque, prisée pour ses propriétés aseptiques. Une fierté particulière est tirée de l'aménagement - aux normes les plus récentes de l'hygiène - de la cuisine, «revêtue de mosaïques avec frise éclatante rappelant le cuivre et l'aluminium de l'outillage et des chambres pour aliments avec mosaïques lavables à la lance ${ }^{33}$ ». Pour réaliser ce chantier très important entre 1936 et 1938, Marcel Rousseau fait appel à l'atelier du mosaïste rennais d'origine italienne, Isidore Odorico. Dans le style Art déco qu'il affectionne, Odorico répond ici à une commande de taille qui s'inscrit dans une série de programmes sociaux qui ont contribué à sa renommée ${ }^{34}$. Des photographies de l'époque et des dessins préparatoires font entrevoir la richesse de ces décors où alternent des frises représentant des animaux, des lambris en camaïeu dégradé et des sols traités en tapis géométriques, fuseaux ou quadrillages. Victimes des transformations contemporaines et de l'indifférence face à un patrimoine trop récent et mal compris, ces décors ont presque totalement disparu. En 2012, seules plusieurs dizaines de mètres linéaires de frises figurant des poissons (fig. 15) ont pu être déposées et sauvegardées ${ }^{35}$. 
Figure 15

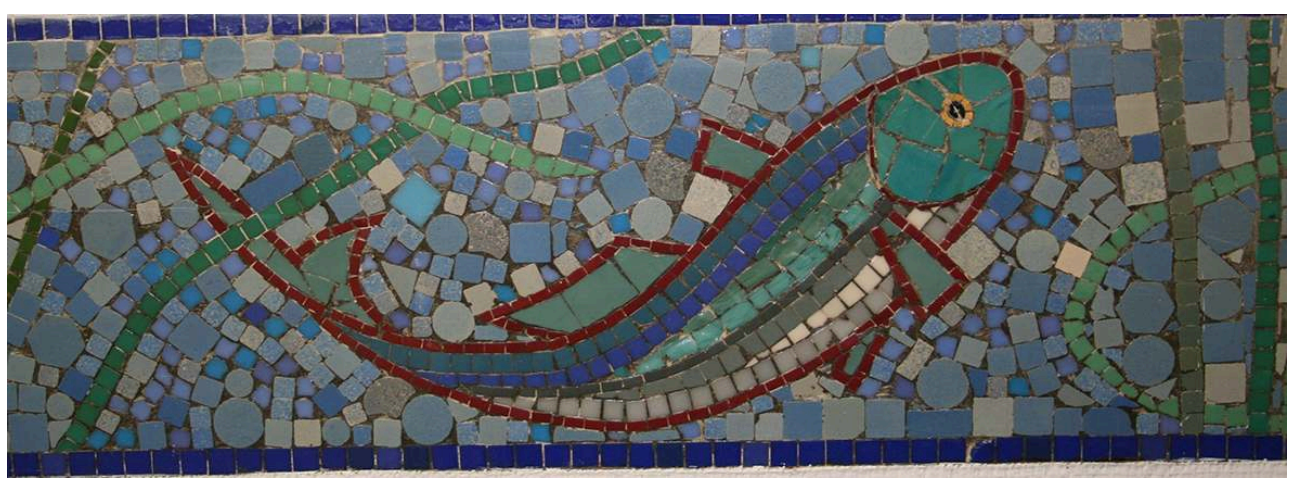

Détail d'une frise de mosaïque d'Odorico figurant plusieurs espèces de poissons. État en 2012.

Phot. Vavasseur, Bernard. (c) Association HeSCO, Saint-Pol-de-Léon.

\section{Le site du Laber}

\section{De la villégiature au préventorium}

En 1920, Perharidy connaît une augmentation de sa capacité d'accueil puisque l'Assistance publique des Hôpitaux de Paris lui confie la gestion d'une grande demeure de villégiature entourée d'un parc de plusieurs hectares, le " château du Laber ", situé à un kilomètre au sud-est du sanatorium. C'est un édifice de style éclectique composé d'une multitude de pavillons, de tours et de tourelles en surplomb (fig. 16). Coiffé de toitures complexes, il avait été construit vers 1890 pour la famille Laurent-Geoffroy, d'après - selon la tradition orale - les plans de l'architecte parisien Charles Girault ${ }^{36}$. Malgré son allure imposante, le bâtiment est peu fonctionnel et encore moins adapté à sa nouvelle destination, celle d'un préventorium pour filles âgées de 4 à 16 ans. Seul le salon orné « d'une grande cheminée d'art marocain et pourvu d'un balcon intérieur ${ }^{37}$ » peut servir de dortoir et accueillir 35 malades en 1920, capacité qui passe à 60 lits en 1935, après des travaux de confort élémentaire tels que l'installation de l'eau, de l'électricité, du chauffage et même la pose d'huisseries (fig. 17). 
Figure 16

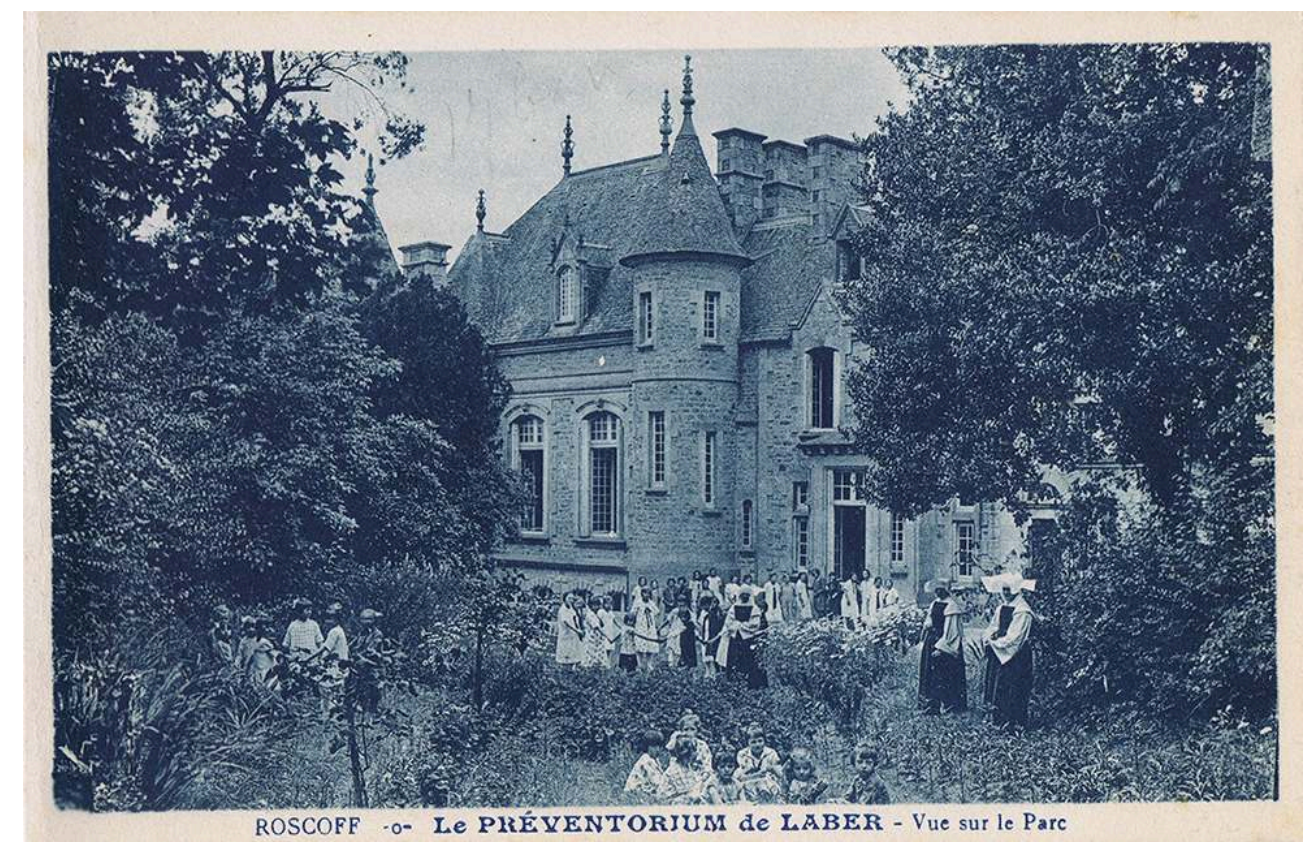

L'ÉlÉVATION SUd et LE PARC dU CHÂteAU dU LABER dANS LES ANNÉES 1930. CARTE POSTALE.

(C) Collection Anquet, Roscoff.

Figure 17

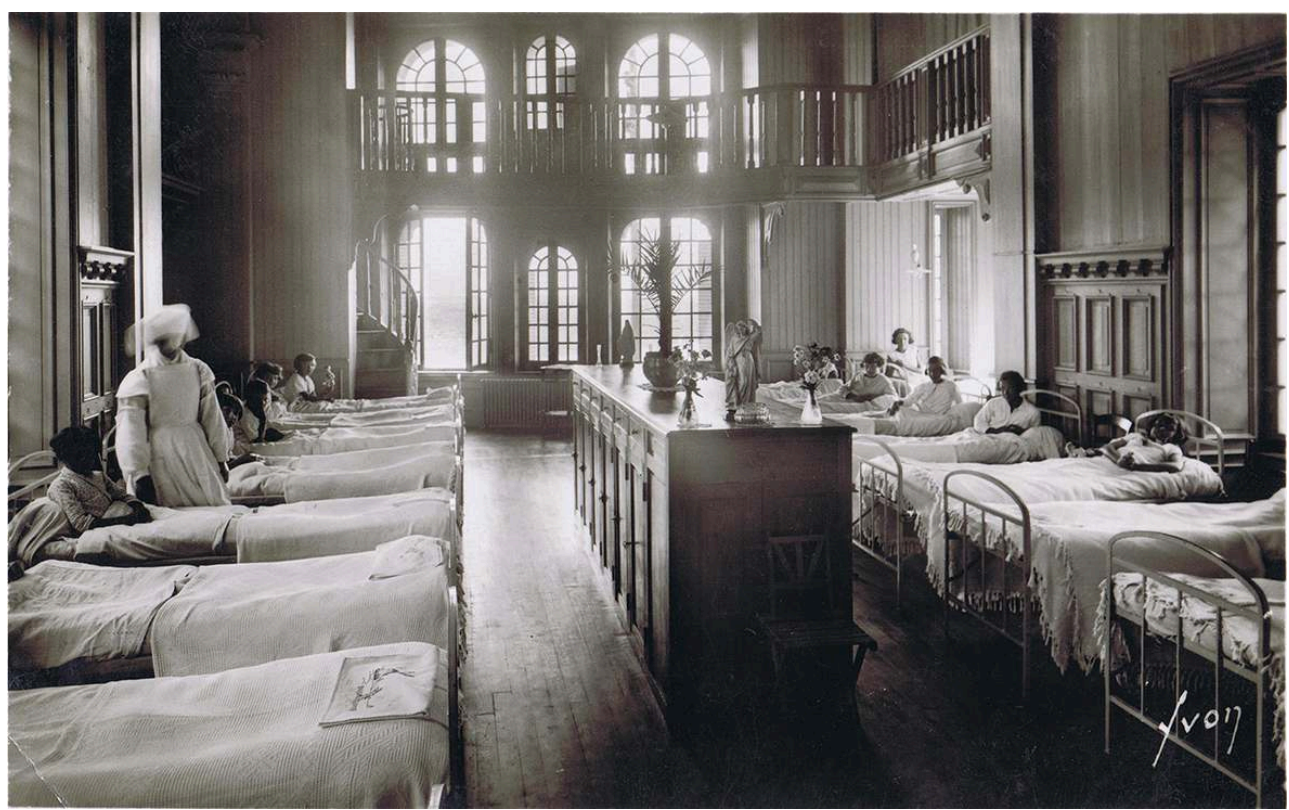

LE CHÂTEAU dU LABER TRANSFORMÉ EN PRÉVENTORIUM dANS LES ANNÉES 1930. LE gRAND SALON SERT DE DORTOIR. CARTE POSTALE.

(c) Association HeSCO, Saint-Pol-de-Léon.

\section{Un sanatorium « moderne »}

40 Afin de répondre à une demande grandissante de lits mais aussi d'assurer des revenus supplémentaires indispensables au fonctionnement du sanatorium et de son annexe le 
Laber, une partie du parc du château est consacrée à l'implantation d'un nouveau bâtiment appelé communément « clinique du Laber ». La première tranche de travaux est réalisée entre 1925 et $1928^{38}$, la seconde entre 1934 et 1936 selon les directives de Marcel Rousseau, à la tête de l'ensemble des institutions. D'une capacité de 100 lits, l'édifice sert de sanatorium - fort confortable - destiné non à des enfants mais à une clientèle de femmes aisées astreintes à des cures d'air sur les galeries ouvertes sur l'extérieur (fig. 18). Conforme aux normes hygiénistes de l'époque, le bâtiment s'inscrit dans la vague de constructions de sanatoriums et de préventoriums d'un nouveau type. Il illustre une conception architecturale liant rationalité distributive et esthétique dépouillée ${ }^{39}$. Sa structure - piliers, poutres et planchers - est en béton armé ${ }^{40}$.

Figure 18

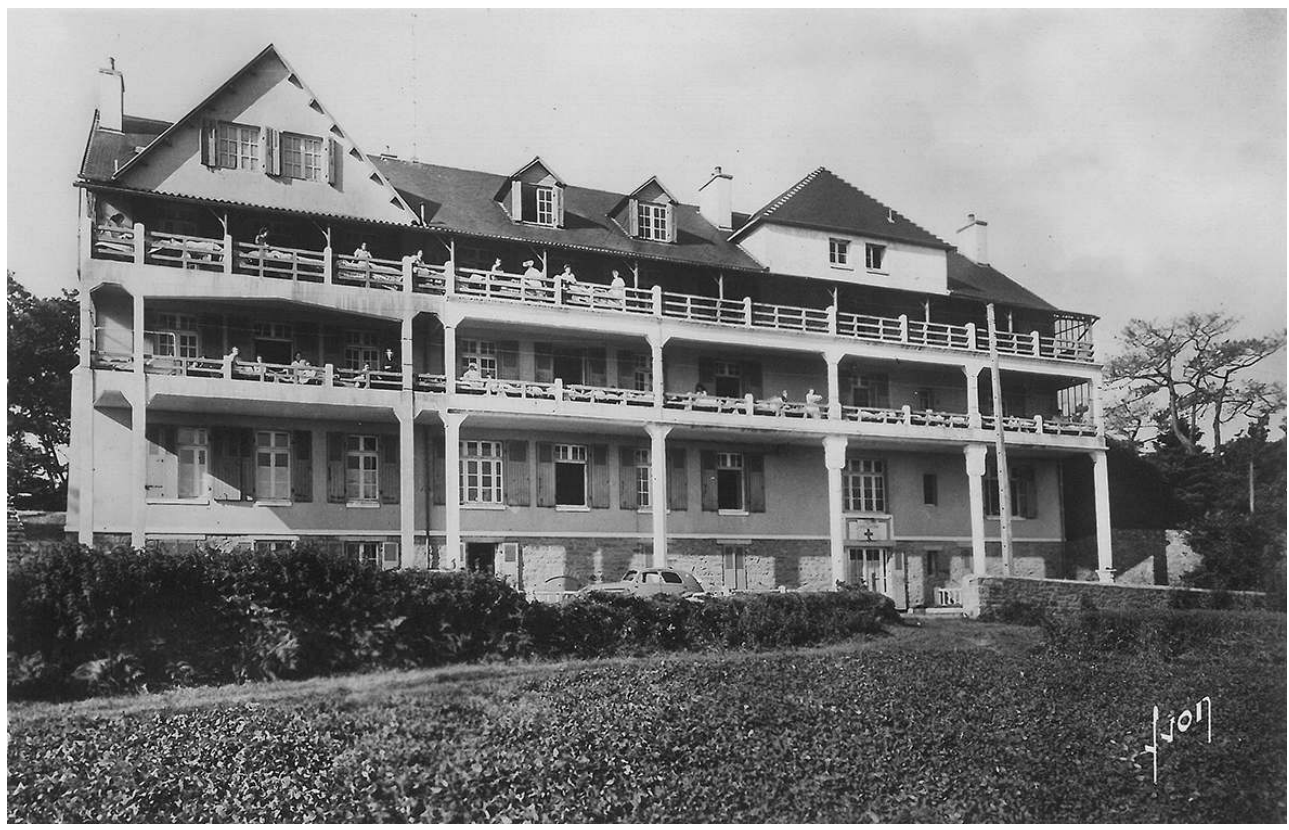

Le nouveau sanatorium dit « clinique du Laber », élévation nord, années 1950. Les trois travées de droite sont achevées en 1927, les deux autres ont été rajoutées en 1934. Des cures d'air identiques existent au sud ; elles ont toutes été obturées dans les années 1980 par des vitrages pour augmenter les surfaces intérieures. Carte postale.

(c) Association HeSCO, Saint-Pol-de-Léon.

\section{Les mosaïques}

41 Un vaste ensemble de mosaïques couvrant une partie des sols et des murs des quatre niveaux du bâtiment a été redécouvert en 2013. Cachées par des revêtements modernes, elles étaient tombées dans l'oubli. Les pièces les plus importantes correspondent aux cuisines réaménagées en 1936. Les couloirs, "également revêtus de mosaïques, ont pris un aspect gai et accueillant ${ }^{41} »$. Fabriquées au même moment et dans un esprit de "politique des mosaïques » semblable à celui du sanatorium pour enfants, leur réalisation est également confiée à l'atelier Odorico de Rennes.

42 Ici aussi, des grès cérame, choisis pour leur résistance, sont utilisés pour les sols et les lambris tandis que des "petits émaux dimensionnés ", notamment des émaux de Briare, composent les frises dans un style inspiré de l'Art déco. En l'absence de motifs figuratifs, certaines frises sont parsemées de pâtes de verre et de smalts (verres colorés) 
d'or ou d'argent (fig. 19). Ces mosaïques témoignent d'une période très féconde de l'atelier Odorico et comptent parmi les plus grands ensembles du mosaïste conservés dans l'ouest de la Bretagne ${ }^{42}$. Elles sont aujourd'hui considérées non seulement comme un patrimoine digne d'intérêt mais aussi comme un atout majeur du bâtiment qui les abrite.

Figure 19

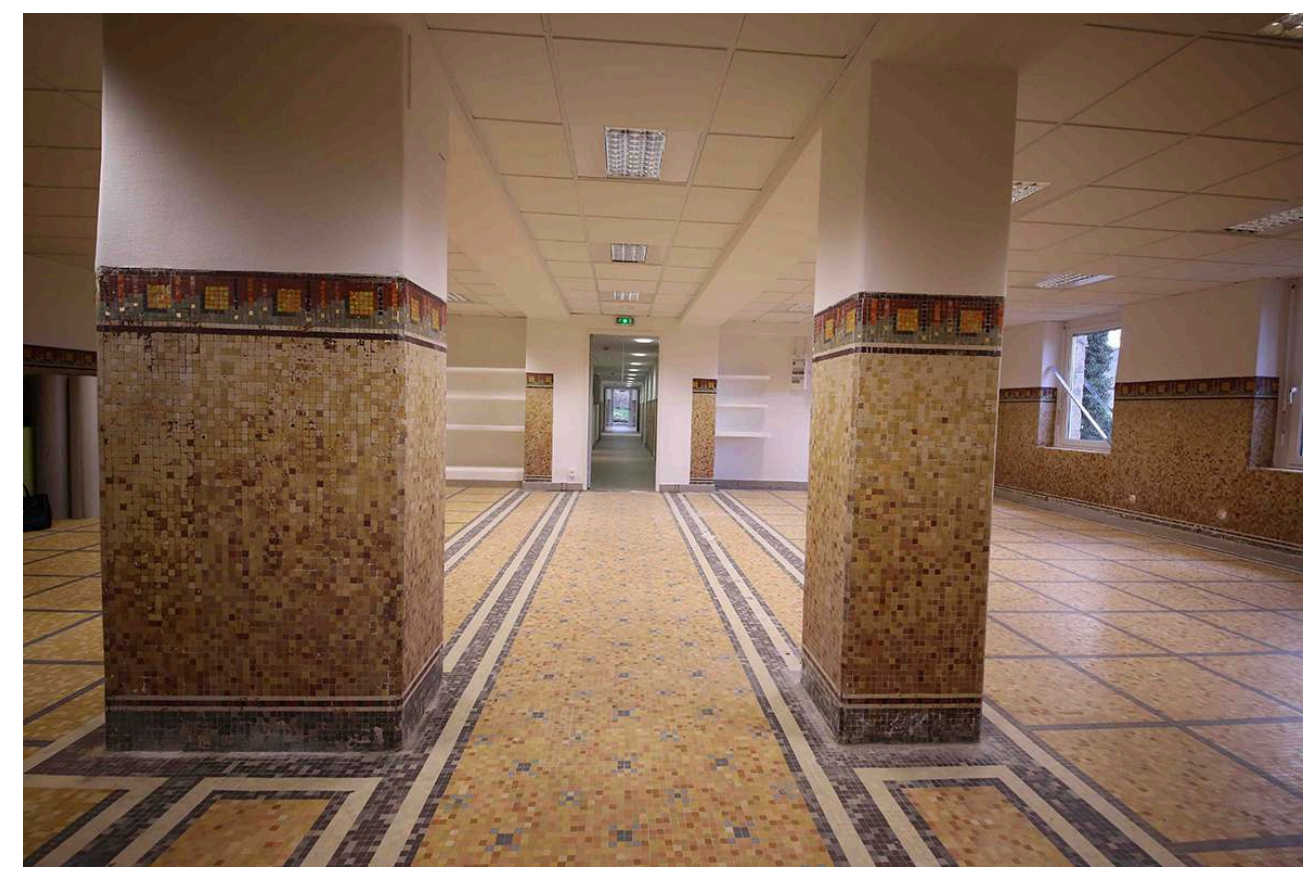

Ancienne « clinique du Laber », salle sud du niveau 0. Revêtement de mosaïque d'Odorico réalisé entre 1937 et 1938. État après réhabilitation, 2015.

Phot. Vavasseur, Bernard. (c) Association HeScO, Saint-Pol-de-Léon.

\section{Conclusion}

Que subsiste-t-il des aménagements d'origine de ces lieux destinés au traitement d'une maladie considérée comme éradiquée dans les années 1960 ? Comme partout en France, dénaturations ou destructions ont largement sévi, jusqu'à une période récente. L'évolution des pathologies nécessitant une évolution des structures hospitalières, tout comme l'indifférence face à un patrimoine insuffisamment reconnu, ont entraîné des pertes importantes. Les bâtiments de Perharidy, peu modifiés jusqu'aux années 1950, témoignaient d'une architecture "normée ", sans accent régional, à l'exception de la chapelle de style néo-breton, restée intacte. Depuis, des restructurations se sont enchaînées entre les années 1970 et aujourd'hui, marquées par des constructions parfois hors gabarit, dans ce site naturellement exigu et donc peu propice aux extensions.

Perharidy est aujourd'hui encore une fondation. Employeuse de 600 personnes, la fondation Ildys gère l'établissement, désormais spécialisé dans les soins de suite et de réadaptation ${ }^{43}$. Si la vocation médicale a été maintenue et pérennisée grâce à la détermination des équipes dirigeantes successives, cela n'a pu se faire sans intervenir sur l'outil de travail, les bâtiments et les équipements atteints d'obsolescence mais que 
nous qualifions aujourd'hui de patrimoine. L'habitude de n'apprécier que le patrimoine traditionnel ou religieux occulte encore le regard porté sur le patrimoine lié à la santé. Comment reconnaître à ce lieu, relatif à la maladie et souvent à la souffrance, des qualités esthétiques intrinsèques, même si les choix faits à certains moments ont su démontrer que fonctionnalité et beauté n'étaient en rien contradictoires mais voulues et bénéfiques?

Figure 20

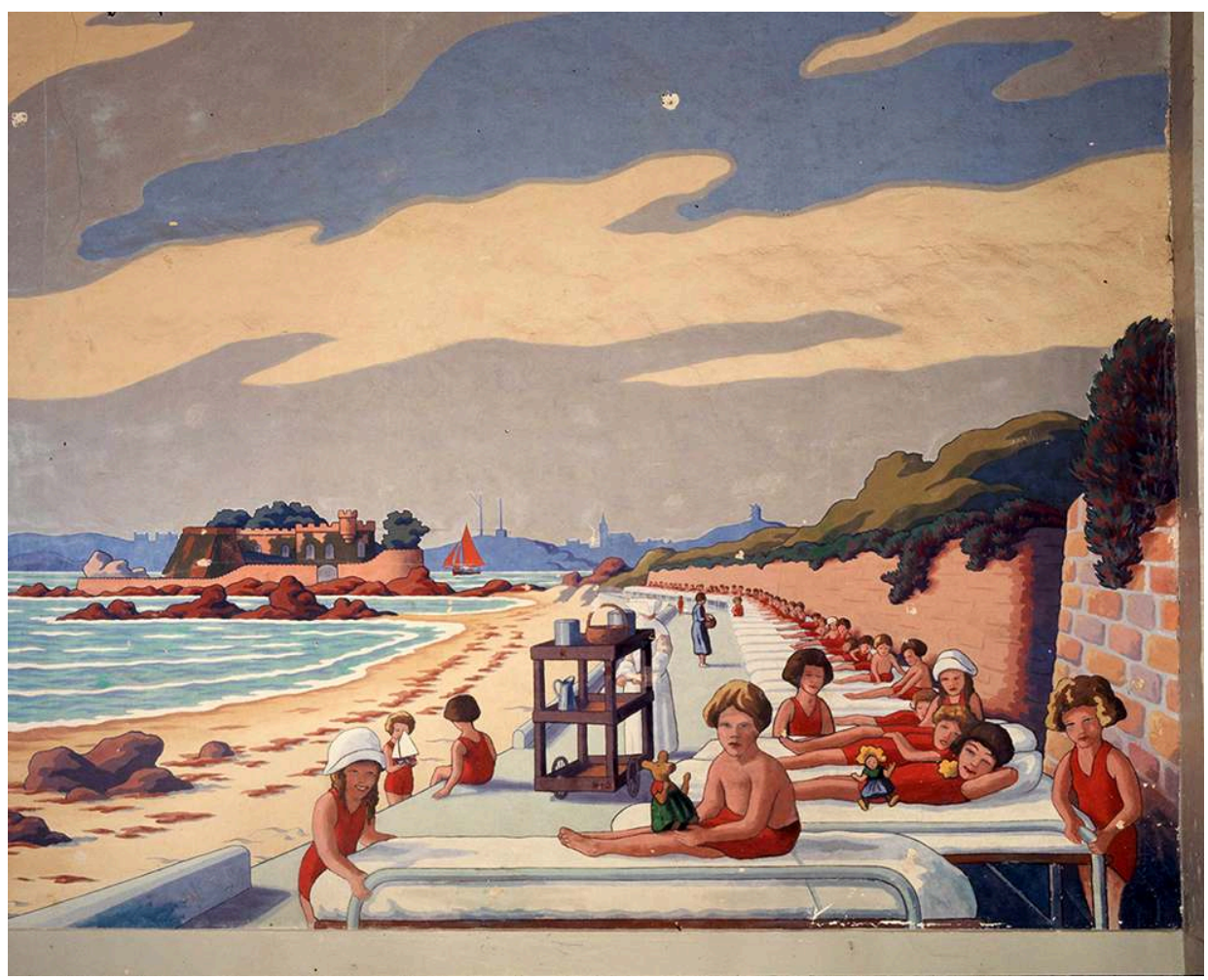

Ancienne salle des fêtes, détail d'une peinture murale réalisée en 1934 par Charles de Kergariou. La cure d'air, l'îlot Jacopin et, au fond, l'île de Batz. État en 1992.

Phot. Artur, Guy. Service de l'inventaire du patrimoine culturel. ( ) Région Bretagne, Rennes.

Cela vaut pour les peintures murales de Charles de Kergariou qui ornaient l'ancienne salle des fêtes (fig. 20) ; recensées dès 1984, défendues et sauvées de la destruction en 1992, puis restaurées, elles sont aujourd'hui exposées dans le gymnase. C'est vrai aussi pour le très grand ensemble de mosaïques de l'atelier Odorico ${ }^{44}$ (plusieurs centaines de mètres carrés) couvrant les sols, les lambris et une partie des soubassements extérieurs mais dont ne subsistent que des vestiges toutefois reconnus comme éléments patrimoniaux avérés (fig. 21). Des frises de poissons ont ainsi été sauvegardées en 2012. Déposées, elles attendent une mise en valeur appropriée et accessible à tous les publics (fig. 22). 
Figure 21

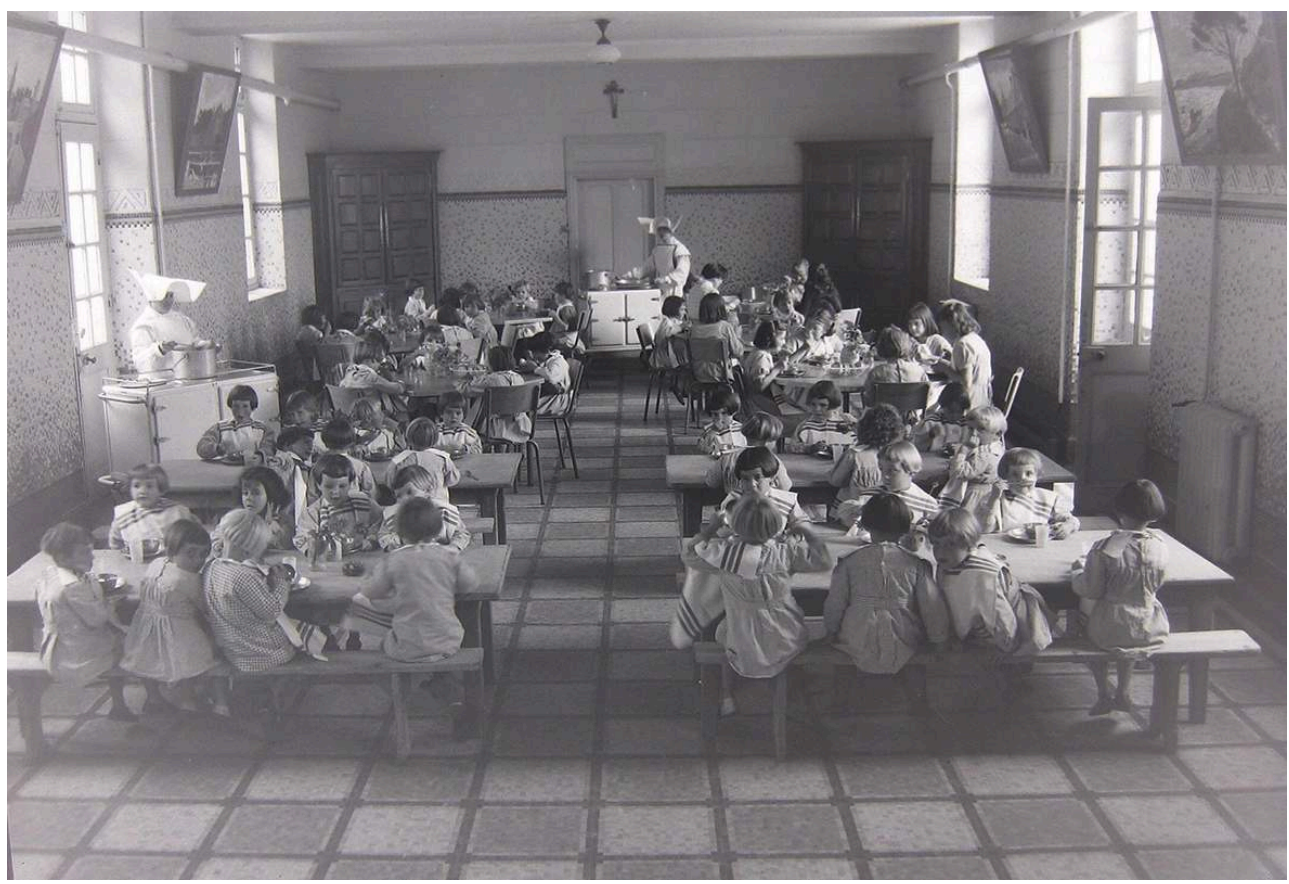

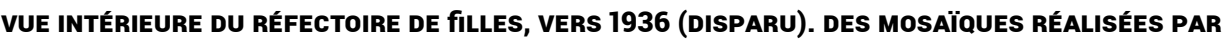
L'ATELIER ODORICO DE RENNES COUVRENT LE SOL ET LES LAMBRIS. CARTE POSTALE.

(C) Collection Studio Piriou, Roscoff.

Figure 22

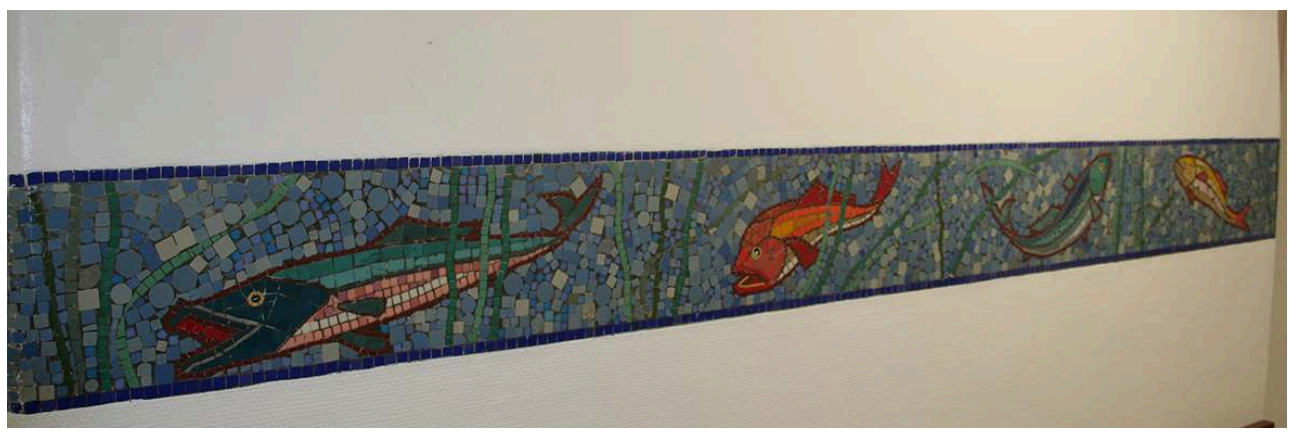

Détail d'une frise de mosaïque d'Odorico figurant des poissons. État en 2012.

Phot. Vavasseur, Bernard. (c) Association HeSCO, Saint-Pol-de-Léon.

Deux projets de valorisation sont actuellement en cours à Perharidy. Une présentation historique et patrimoniale au moyen d'outils de médiation numériques: des $Q R$ codes, codes-barres gravés sur un support près des éléments patrimoniaux qu'ils décrivent et dont la lecture se fait au moyen de téléphones cellulaires ou de tablettes, seront en place pour l'été 2016. Ce dispositif, particulièrement adapté aux diverses contraintes rencontrées dans un établissement de santé en activité, a été sélectionné dans le cadre "Culture-Santé 2015 », protocole régional interministériel piloté par le ministère de la Culture (Drac Bretagne).

47 Le deuxième projet concerne, à moyen terme, la réhabilitation de la cure d'air, espace fréquenté par un public nombreux - patients, personnel, visiteurs - et dont la configuration se prête à la présentation des frises de mosaïques sauvegardées en 2012, 
d'autant qu'il jouxte ici un site naturel de grande qualité géré par le Conservatoire du littoral ${ }^{45}$.

Quant à l'ensemble du site du Laber (château, ancienne clinique et parc), il n'est plus géré par Perharidy depuis 2012, date à laquelle il est devenu propriété de la communauté de communes du Pays Léonard. La clinique qui avait été reconvertie en centre de réadaptation alimentaire pour adolescents en 1981, puis définitivement fermée en 2007, a fait l'objet d'une réhabilitation en 2013. Elle abritera désormais des salles de cours et des logements destinés aux étudiants de la station biologique de Roscoff-CNRS, nouvelle affectation qui s'inscrit dans la vocation de Roscoff, ville tournée vers la mer, la recherche et la santé.

Figure 23

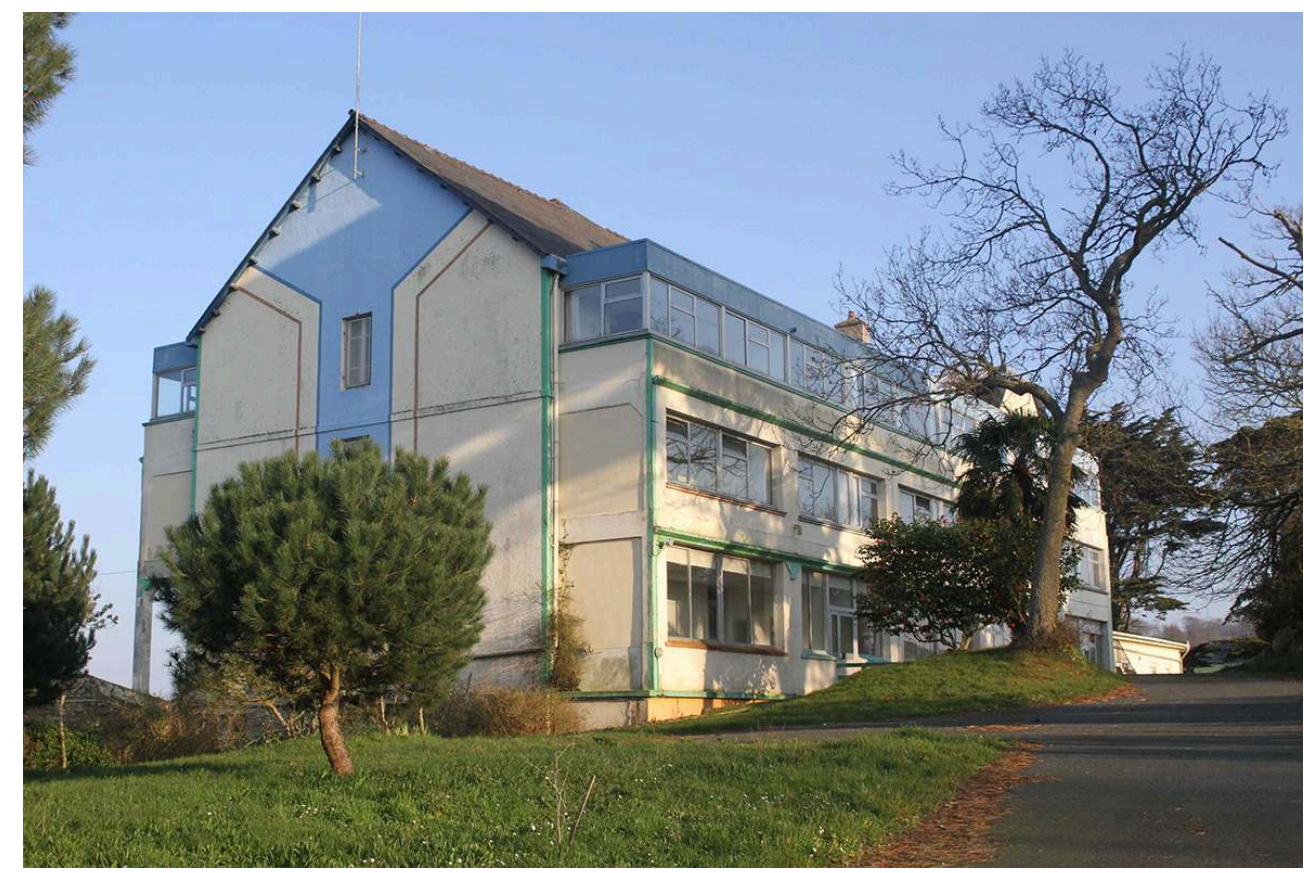

Élévation sud de l'ancienne « clinique du Laber » en cours de réhabilitation.

Phot. Vavasseur, Bernard. (c) Association HeScO, Saint-Pol-de-Léon.

Si l'on porte aujourd'hui, grâce à l'intervention de l'association HeSCO, un regard neuf sur ce bâtiment que certaines modifications, notamment l'obstruction des anciennes cures d'air par des vitrages modernes, ont passablement dénaturé, il demeure que la morphologie, les distributions intérieures et la structure même de l'édifice, composé de piliers, de poutres et de planchers en BAH (béton armé Hennebique) n'ont pas été affectées (fig. 23). Son intérêt patrimonial est amplifié par la présence des mosaïques redécouvertes puis sauvées par l'association HeSCO au moment des travaux de réhabilitation. Aujourd'hui, les mosaïques de deux grandes pièces affectées à des salles de cours, ainsi que celles du couloir central du niveau 0, sont en grande partie apparentes (fig. 24). Plusieurs autres pièces également décorées n'ont toutefois pu échapper au cloisonnement et à la pose de revêtements muraux modernes. 
Figure 24

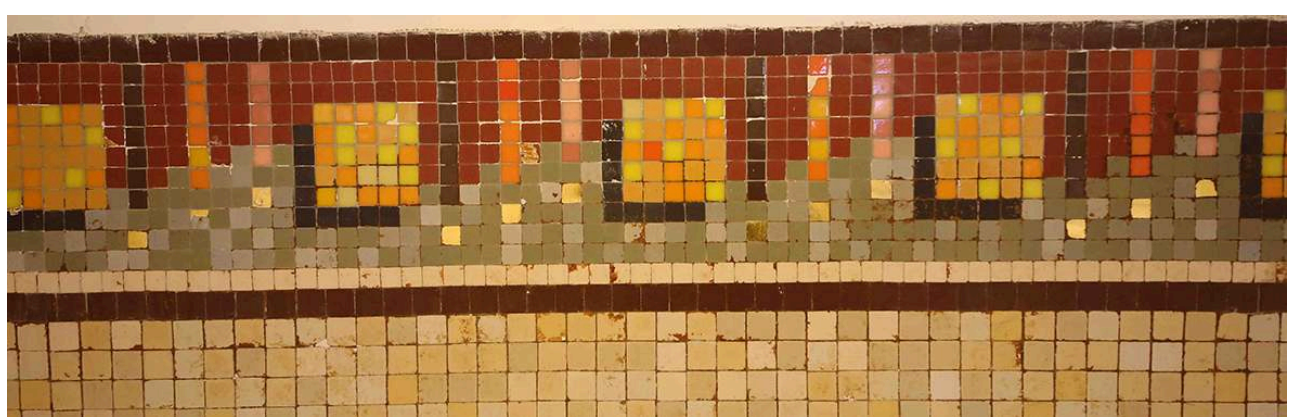

Ancienne « clinique du Laber », salle sud du niveau 0. Détail d'une frise d'Odorico réalisée entre 1937 et 1938. État après réhabilitation, 2015.

Phot. Vavasseur, Bernard. (c) Association HeScO, Saint-Pol-de-Léon.

\section{NOTES}

1. - RUSSEL, Richard. Glandular Diseases, or a Dissertation on the Use of Sea-Water in the Affections of the Glands, 1750. Voir le site: https://en.wikipedia.org/wiki/Richard_Russell_\%28doctor\%29 [consulté le 02/11/2016]. http://www.mybrightonandhove.org.uk/page_id__8719.aspx [consultés le $02 / 11 / 2016]$.

2. - En Bretagne, d'autres établissements maritimes destinés aux soins antituberculeux, mais pas spécialement dédiés aux enfants, sont créés plus tardivement. C'est le cas des préventoriums de Saint-Laurent-de-la-Mer à Plérin (1924-1926) et de Trestel à Trévou-Tréguignec (1929-1936) dans les Côtes-d'Armor et surtout du grand sanatorium départemental de Kerpape (1920) à Ploemeur près de Lorient (Morbihan). Le très beau projet proposé en 1906 par l'architecte Auguste Perret pour le sanatorium de Trélévern (Côtes-d'Armor) ne verra pas le jour. Voir: http:// 
www.citechaillot.fr/ressources/expositions_virtuelles/vegetal/03-theme05-sstheme04doc01.html [consulté le 02/11/2016].

3. - Intervention du Docteur MORAND, directeur du sanatorium départemental de Plougonven. Dans: Actes du Congrès de l'Alliance d'hygiène sociale, Quimper, 5-6 octobre 1929. Paris, 1929. Consultable sur http://gallica.bnf.fr/ark:/12148/bpt6k57190638/f21.image.r=Plougonven.langFR [consulté le 02/11/2016].

4. - Banyuls-sur-Mer. Le sanatorium: vue générale (gravure). Paris, bibliothèque interuniversitaire de santé (BIU). http://www.biusante.parisdescartes.fr/images/banque/pt/ CISB0576.jpg [consulté le 02/11/2016]. Les sanatoriums de Banyuls et de Perharidy se ressemblent : même site maritime isolé, plan général comparable.

5. - Station biologique de Roscoff /Centre de recherche et d'enseignement en biologie et écologie marines, université de Paris/CNRS. Voir: TOULEMOND, André. «La station biologique. Un lieu voué à la science ». Dans : DOUARD, Christel et GUESNIER, Valérie. Recherche, santé et patrimoine à Roscoff. Rennes : Éditions Apogée (Itinéraire de découverte), 2014, p. 14-17.

6. - Louis Bagot (1862-1941), médecin généraliste formé à l'École de médecine navale de Brest, passionné de zoologie, de physiologie et de climatologie, avait installé en 1899 à Roscoff un cabinet secondaire spécialisé dans le traitement des rhumatismes par l'eau de mer.

7. - Les archives publiques conservent peu de documents. Les archives privées de Perharidy étaient malheureusement inaccessibles lors de la rédaction de cette contribution. Elles contiennent vraisemblablement des éléments importants pour affiner davantage l'évolution historique, médicale, organisationnelle et patrimoniale de l'établissement.

8. - Extraits du courrier du docteur Albert Calmette à H. de Fontenilliat, 1928 : « (...) Je connais la plupart des établissements privés qui, de Zuydcoote à Hendaye et de Banyuls-sur-Mer aux Îles d'Or, se sont multipliés depuis moins d'un siècle parce que il a bien fallu se rendre à l'évidence et se convaincre que le séjour prolongé au bord de la mer, l'air chargé d'iode et les vents salés du large représentent à eux seuls la thérapeutique la plus efficace que nous connaissions pour guérir les tuberculoses des ganglions, pour aider la chirurgie à guérir celles des articulations ou des os, malheureusement si fréquentes parmi les adolescents et les enfants d'âge scolaire dans nos familles françaises. Plusieurs de ces sanatoriums marins rendent d'immenses services et leurs fondateurs sont justement fiers des résultats qu'ils en obtiennent. Mais j'ose affirmer qu'aucun ne peut supporter la comparaison avec celui de Roscoff (...). Je ne crois pas qu'on eût pu choisir un emplacement plus favorable que cette presqu'île de Perach'idy (sic) protégée contre la grosse mer du large par l'île de Batz et baignée par les eaux tièdes du Gulf-Stream.Peu à peu, et vous me permettrez bien d'ajouter grâce à votre belle énergie, à votre si claire compréhension de ce que doit être une telle œuvre, le succès s'est affirmé. Le nombre est déjà considérable des enfants devenus des pères ou des mères de familles, qui doivent au Sanatorium de Roscoff d'avoir recouvré la santé. Ce sanatorium, dont vous avez préparé et assuré le développement, est aujourd'hui, à mon avis, par sa situation, par sa tenue, par son outillage, par la compétence et le dévouement de son personnel technique - religieuses, infirmières, médecins - le plus parfait qui existe en France ». Arch. dép. du Finistère, Q 4 BB 1178. Le sanatorium de Roscoff et ses annexes. Brochure de présentation pour le cinquantième anniversaire de l'établissement, 1951.

9. - Arch. dép. du Finistère, sans cote. Le Lien. Bulletin trimestriel des amis, des anciens, des malades du sanatorium de Roscoff, 1935-1958.

10. - Le sanatorium de Perharidy avait alors reçu de l'Assistance publique des Hôpitaux de Paris le site du Laber en gestion.

11. - CREMNITZER, Jean-Bernard. Architecture et santé. Le temps du sanatorium en France et en Europe. Paris : Picard, 2005.

12. - DELECOURT, Nicolas. 1901-2001. Du Sanatorium marin de Roscoff au Centre de Perharidy. Roscoff: fondation Centre héliomarin de Roscoff, 2001 (réédition 2011). 
13. - Arch. dép. du Finistère, Q 4 BB 1178. Le sanatorium de Roscoff et ses annexes. Brochure de présentation pour le cinquantième anniversaire de l'établissement, 1951.

14. - Arch. dép. du Finistère, 1 X 149. Extrait du procès-verbal des délibérations du conseil général du Finistère, 13 septembre 1916.

15. - Arch. dép. du Finistère, sans cote. Le Lien. Bulletin trimestriel des amis, des anciens, des malades du sanatorium de Roscoff, 1935-1958.

16. - Arch. dép. du Finistère, 1 X 149. Extrait du procès-verbal des délibérations du conseil général du Finistère, 13 septembre 1916.

17. - Dr. LUCAS. Rapport sur les dispensaires, baraquements et sanatoriums, 1916. Se met alors en place le projet d'un sanatorium départemental pour soigner les Finistériens atteints de tuberculose pulmonaire, dans un lieu pas trop éloigné de leur domicile puisque l'idée de faire soigner les malades dans une autre région au climat plus propice (moyenne et haute montagne) n'est pas envisageable car «le climat n'est nullement préjudiciable au traitement de la tuberculose lorsqu'il s'agit du Breton, qui aime la pluie et qui y est habitué (...). Nous nous heurtons à la répugnance instinctive de nos Bretons à s'expatrier hors du Finistère (...). La nature même du Breton ne permet pas de songer à le diriger d'office sur un sanatorium qui serait éloigné de son pays (...). Vous soignerez le Breton chez lui, dans l'établissement créé dans le Finistère, ou vous ne le soignerez pas ». L'établissement ouvre ses portes en 1919 au sud de Morlaix, à Guervenan, commune de Plougonven. Arch. dép. du Finistère, 1 X 149. Extrait du procès-verbal des délibérations du conseil général du Finistère, 13 septembre 1916.

18. - Un Sanatorium marin à Roscoff. voir : Centre National du Cinéma/BnF. Paris : http://www.cncaff.fr/internet_cnc/Fiches/Oeuvre/ResultatRechercheSimple.aspx [consulté le 02/11/2016].

19. - L'architecture des sanatoriums a récemment donné lieu à d'importants travaux de recherche. Voir : GRANDVOINNET, Philippe. Architecture thérapeutique : histoire des sanatoriums en France (1900-1945). Genève : Métis Presses, 2014.

20. - Joseph-Charles de Montarnal (1867-1947) réalise un grand nombre d'immeubles à Paris et les environs. Voir : STRUCTURAE. Base de données internationale du patrimoine du génie civil. http://structurae.info/personnes/joseph-charles-de-guirard-de-montarnal [consulté le 02/11/2016].

21. - La Maison Saint-Luc est édifiée entre 1900 et 1904 à la demande de la congrégation des Filles du Saint-Esprit afin de servir de lieu de séjour aux patients traités à l'Institut marin du Dr. Bagot. Contemporaine aux débuts de l'hydrothérapie à Roscoff, la Maison Saint-Luc semble être la réalisation la plus importante de l'architecte en Bretagne.

22. - Seule une reproduction de ce document était disponible. Voir: DELECOURT, Nicolas. 1901-2001. Du Sanatorium marin de Roscoff au Centre de Perharidy. Roscoff: fondation Centre héliomarin de Roscoff, 2001 (rééd. 2011), p. 11.

23. - Arch. dép. Finistère, sans cote. Le Lien. Bulletin trimestriel des amis, des anciens, des malades du sanatorium de Roscoff, 1935-1958.

24. - Ibid.

25. - Le "petit hôpital maritime " de Berck construit en 1862 suit un plan semblable, à cour fermée, tout comme l'établissement de Pen Bron à La Turballe construit entre 1896 et 1907 dans un style néo-régionaliste par l'architecte Georges Lafont (voir: "L'invention du sanatorium ». Dans : L'Hôpital en France. Histoire et architecture. LAGET, Pierre-Louis, LAROCHE, Claude, DUHAU, Isabelle, (dir.). Lyon : Lieux Dits (« Cahiers du Patrimoine », ${ }^{\circ}$ 99), 2012, p. 333-373. Voir aussi : LUCHIER, Sophie, LAGET, Pierre-Louis, FAURE, Julie et al. Berck-sur-Mer, du soin à la villégiature. Lyon : Lieux Dits (Images du patrimoine, 288), 2014.

26. - La chapelle dédiée à saint Luc est inaugurée en 1923 par l'évêque de Quimper et de Léon, Mgr Duparc. La cloche porte l'inscription : « Te Deum laudamus. Je m'appelle Louise-Marie. Je fus baptisée à Pâques 1923. Ma marraine est Mme de Fontenilliat, nièce et continuateur de la marquise de Kergariou qui fonda le sanatorium. Je sonnerai pour celles qui se dévouent, pour 
ceux qui souffrent et espèrent ». Arch. dép. Finistère, sans cote. Le Lien. Bulletin trimestriel des amis, des anciens, des malades du sanatorium de Roscoff, 1935-1958.

27. - À côté de bâtiments publics et privés, l'architecte Lionel Heuzé réalise dans les années 1920 plusieurs chapelles dont celle de Notre-Dame-de-Lourdes à Primel-Trégastel en Plougasnou (Finistère), quasiment identique à celle de Perharidy.

28. - Marcel Rousseau (1892-1992) participe à la reconstruction de la ville de Reims après la Première Guerre mondiale. Il y construit entre 1922 et 1923 plusieurs immeubles ainsi que deux édifices exceptionnels, le «Cinéma-Opéra » et le "Comptoir de l'Industrie » (voir: http:// www.culture.gouv.fr/culture/dp/patrimoine-xx/pages/1000/frm-hom.htm [consulté le 02/11/2016]). Son logement de fonction à Perharidy a été profondément remanié et abrite aujourd'hui un lieu de recherche médicale (ManRos Therapeutics) appelé hôtel de Recherche.

29. - Arch. dép. Finistère, 1 X 149. Extrait du procès-verbal des délibérations du conseil général du Finistère, 13 septembre 1916.

30. - Kerga (1899-1956). Le peintre de la baie de Morlaix. Cat. expo. Morlaix, musée des Jacobins, 8 avril-26 juin 1994. Morlaix : musée des Jacobins, 1994.

31. - Le service régional de l'Inventaire Bretagne (direction régionale des Affaires culturelles) a réalisé une notice et une couverture photographique de cet ensemble avant sa dépose. Voir dans la base Palissy : notice IM29000970.

32. - Arch. dép. Finistère, sans cote. Le Lien. Bulletin trimestriel des amis, des anciens, des malades du sanatorium de Roscoff, 1935-1958. On recouvre également de mosaïques (non figuratives) les trottoirs et les soubassements extérieurs.

33. - Arch. dép. Finistère, Q 4 BB 1178. Le sanatorium de Roscoff et ses annexes. Brochure de présentation pour le cinquantième anniversaire de l'établissement, 1951.

34. - Les travaux d'Hélène Guéné réalisés en 1983 sur Odorico font entrevoir l'importance des décors de Perharidy, encore visibles en grande partie à cette date. Voir : GUÉNÉ, Hélène. Odorico mosaïste. La production d'un atelier italien en Bretagne et Anjou (1882-1978). Thèse de doctorat, Histoire et Étude des Arts. Université de Haute-Bretagne (Rennes), 1983. Voir aussi : GUÉNÉ, Hélène. Odorico, mosaïste art déco. Bruxelles : Archives d'architecture moderne, 1991 (rééd. 2000). Le musée de Bretagne à Rennes conserve un grand nombre de projets et de dessins préparatoires d'Odorico pour Perharidy. Mosaïste issu d'une dynastie d'artisans italiens, Isidore Odorico (1893-1945) prend la succession de son père après une formation à l'école des beaux-arts de Rennes. L'entreprise réalise alors un grand nombre de décors à mosaïques, aussi bien dans des bâtiments publics que privés, essentiellement dans l'ouest de la France.

35. - C'est à l'association HeSCO (Héritage scientifique et culturel de l'Ouest) qui poursuit depuis plusieurs années l'inventaire, l'étude et la valorisation du patrimoine lié à la science et à la santé de Roscoff, que revient la redécouverte de ces mosaïques.

36. - Il s'agirait de l'unique réalisation de Charles Girault en Bretagne ; architecte prolifique, il est célèbre pour avoir conçu, à Paris, le Petit Palais (1900) et la chapelle funéraire de Pasteur (Paris, Institut Pasteur, 1895). Au Laber, on retrouve sa prédilection pour l'éclectisme, style qui mélange des formes architecturales et décoratives héritées d'époques différentes.

37. - Hélène de Fontenilliat à propos du château : «L'architecte, homme éminent qui fit de très belles choses (...), a prodigué une débauche de tourelles, de clochetons, d'encadrements (...). On retrouve partout, bien inutilement, ce beau granit taillé à l'intérieur, autour des portes, dans les nombreuses antichambres, en marches (...). Un magnifique salon, vraie lanterne entourée de hautes fenêtres, est pourvu d'un balcon intérieur où devaient se tenir des musiciens (...), il a aussi une grande cheminée d'art marocain dont les dorures ne se sont pas ternies depuis trente-sept ans (...). Les chambres du deuxième étage n'avaient pas de fenêtres, celles du premier étaient toutes petites et impossibles à modifier, car elles étaient séparées par des murs comme ceux qu'on faisait du temps des châteaux forts, les escaliers, très nombreux, ne menaient pas tous à 
quelque chose ». Arch. dép. Finistère, sans cote. Le Lien. Bulletin trimestriel des amis, des anciens, des malades du sanatorium de Roscoff, 1935-1958.

38. - L'inauguration de la clinique a lieu en 1928. On y trouve des «chambres avec couleurs différentes ouvrant sur des cures d'air, des couloirs jaunes et une salle à manger bretonne». Arch. dép. Finistère, sans cote. Le Lien. Bulletin trimestriel des amis, des anciens, des malades du sanatorium de Roscoff, 1935-1958.

39. - La clinique du Laber peut se comparer à deux établissements à peu près contemporains et stylistiquement semblables: le sanatorium de Trestel à Trévou-Tréguignec (Côtes-d'Armor) : http://patrimoine.bzh/gertrude-diffusion/dossier/sanatorium-dit-centre-de-reeducation-et-dereadaptation-fonctionnelles-de-trestel/17876f09-408d-4d7b-a7e7-92fda7ef0136 et le sanatorium départemental de Kerpape à Ploemeur près de Lorient (Morbihan) : http://www.ouest-france.fr/ au-fil-de-lhistoire-les-cent-ans-de-kerpape-2572609 [consultés le 02/11/2016].

40. - Marcel Rousseau est mentionné comme commanditaire des travaux. Voir: Fonds Bétons armés Hennebique (BAH), Subdiv. 10 : Bretagne. 076 IFA. Objet BAH-06-1934-49093. Sanatorium marin, Roscoff et Santec (Finistère), transformations. 1934-1952. http://archiwebture.citechaillot.fr/fonds/FRAPN02_BAH10/inventaire[consulté le 02/11/2016].

41. - Arch. dép. Finistère, sans cote. Le Lien. Bulletin trimestriel des amis, des anciens, des malades du sanatorium de Roscoff, 1935-1958.

42. - Le musée de Bretagne à Rennes conserve quelques dessins préparatoires d'Odorico pour la clinique du Laber. Voir : GUÉNÉ, Hélène. Odorico mosaïste. Op. cit., vol. 2, p. 347.

43. - Prise en charge des patients porteurs de maladies graves, de séquelles d'accidents, de maladies chroniques essentiellement à conséquence nutritionnelle, dialyse, réadaptation fonctionnelle, réinsertion familiale et socioprofessionnelle. Voir: http://www.ildys.org/-lafondation [consulté le 02/11/2016].

44. - DOUARD, Christel, GUESNIER, Valérie, PUGET, Catherine. Un littoral enchanté. Le décor de Kerga au sanatorium de Roscoff. Lopérec : Éditions Locus Solus, 2016.

45. - Le Conservatoire du littoral, propriétaire depuis 2010 de cinq hectares situés à la pointe nord de la presqu'île de Perharidy, a aménagé en 2015 un parcours pour piétons permettant de profiter de la beauté du site. Voir : http://www.conservatoire-du-littoral.fr/siteLittoral/407/28pointe-de-perharidy-29_finistere.htm [consulté le 02/11/2016].

\section{RÉSUMÉS}

En Bretagne, le patrimoine lié à la santé demeure largement méconnu. Et pourtant, la région est riche d'éléments originaux qui, apparus aux $\mathrm{XIX}^{\mathrm{e}}$ et $\mathrm{xx}^{\mathrm{e}}$ siècles, éclairent les liens qui existent entre les mentalités, l'aménagement du territoire, les découvertes scientifiques, les maladies, l'architecture ou encore la création artistique. Cet article mettra l'accent sur un grand sanatorium héliomarin né, à l'instar d'une série d'établissements du même type, sur le littoral français au début $\mathrm{du} \mathrm{xx}^{\mathrm{e}}$ siècle. Grâce au mécénat privé, les premiers bâtiments du sanatorium pour enfants atteints de tuberculose osseuse de Roscoff sortent de terre en 1901. Par la suite, plusieurs architectes font évoluer les bâtiments. Les années 1930 sont marquées par la modernisation intérieure et la mise en place de décors partiellement conservés. L'annexion d'une vaste demeure de villégiature de la fin du XIX siècle servant de préventorium et la construction d'un autre sanatorium durant l'Entre-deux-guerres complètent le plus important établissement 
de prévention et de soins antituberculeux du département du Finistère. Chercheurs, médecins, architectes, artistes, philanthropes, célèbres ou œuvrant dans l'ombre, ont contribué à façonner le site et laissé un patrimoine aujourd'hui fragile et menacé.

In Northern Brittany, the health-related heritage remains largely unknown, despite the fact that this region can count many original establishments, built in the late nineteenth or early twentieth century and underlining the links between attitudes, town and country planning, scientific discoveries, illnesses, architecture or even artistic creation. This paper aims to highlight the role of a big seaside treatment hospital using heliotherapy which was, like other similar establishments, founded in the early twentieth century. Thanks to private endowments, the first buildings devoted to children suffering from bone tuberculosis were erected in 1901 . Later, several architects transformed the original buildings. The 1930s brought modernisation and internal decoration, some of which is partly still in place. The addition of a large private manor house built in the late nineteenth century as an observation sanatorium and the building of a new sanatorium in the inter-war years completed the largest institution devoted to the prevention and the cure of tuberculosis in Northern Brittany. Researchers, doctors, architects, artists, philanthropists, whether they be famous or working in the shade, all contributed to the shaping of the site, leaving a heritage which is today fragile and endangered.

\section{INDEX}

Keywords : Roscoff, Perharidy, Roscoff biological station, Roscoff marine institute, Bagot (Louis), Kergariou (Louise de), Calmette (Albert), Fontenilliat (Hélène de), Montarnal (Joseph-Charles de), Heuzé (Lionel), fresh air therapy, Kergariou (Charles de), Odorico, Girault (Charles), Rousseau (Marcel)

Mots-clés : Roscoff, Perharidy, station biologique de Roscoff, Institut marin de Roscoff, Bagot (Louis), Kergariou (Louise de), Calmette (Albert), Fontenilliat (Hélène de), Montarnal (JosephCharles de), Heuzé (Lionel), cure d'air, Kergariou (Charles de), Odorico, Girault (Charles), Rousseau (Marcel)

\section{AUTEURS}

\section{CHRISTEL DOUARD}

Ingénieur d'études. Service de l'inventaire du patrimoine culturel, région Bretagne christel.douard@orange.fr

\section{AVEC LA COLLABORATION DE VALÉRIE GUESNIER}

Association Hesco et chargée de mission à l'action culturelle pour Haut-Léon communauté office de tourisme Valerie.guesnier@aliceadsl.fr 